\title{
Short Timescale Core Dynamics Theory and Observations
}

\section{Conference Paper}

\section{Author(s):}

Finlay, C.C.; Dumberry, M.; Chulliat, A.; Pais, M.A.

Publication date:

2010-08

Permanent link:

https://doi.org/10.3929/ethz-b-000159315

\section{Rights / license:}

In Copyright - Non-Commercial Use Permitted

\section{Originally published in:}

Space Science Reviews 155(1-4), https://doi.org/10.1007/s11214-010-9691-6 


\title{
Short Timescale Core Dynamics: Theory and Observations
}

\author{
C.C. Finlay • M. Dumberry • A. Chulliat • M.A. Pais
}

Received: 22 March 2010 / Accepted: 23 August 2010 / Published online: 20 October 2010

(C) Springer Science+Business Media B.V. 2010

\begin{abstract}
Fluid motions in the Earth's core produce changes in the geomagnetic field (secular variation) and are also an important ingredient in the planet's rotational dynamics. In this article we review current understanding of core dynamics focusing on short timescales of years to centuries. We describe both theoretical models and what may be inferred from geomagnetic and geodetic observations. The kinematic concepts of frozen flux and magnetic diffusion are discussed along with relevant dynamical regimes of magnetostrophic balance, tangential geostrophy, and quasi-geostrophy. An introduction is given to free modes and waves that are expected to be present in Earth's core including axisymmetric torsional oscillations and non-axisymmetric Magnetic-Coriolis waves. We focus on important recent developments and promising directions for future investigations.
\end{abstract}

Keywords Geomagnetism · Secular variation · Core dynamics · Core-mantle coupling

\section{Introduction}

It has been long recognized that the Earth's magnetic field is not steady. The first reported observation of temporal field changes dates back to 1635, when Gellibrand (1635) noted a

C.C. Finlay $(\bowtie)$

Institut für Geophysik, ETH Zürich, 5 Sonneggstrasse, Zürich, 8092, Switzerland

e-mail: cfinlay@erdw.ethz.ch

M. Dumberry

Department of Physics, University of Alberta, Edmonton, AB, T6G 2G7, Canada e-mail: dumberry@phys.ualberta.ca

A. Chulliat

Equipe de Géomagnétisme, Institut de Physique du Globe de Paris, 4, Place Jussieu, 75252,

Paris Cedex 05, France

e-mail: chulliat@ipgp.jussieu.fr

M.A. Pais

Physics Department, University of Coimbra, 3004-516 Coimbra, Portugal

e-mail: pais@ fis.uc.pt 
change in the magnetic declination at London. Nowadays, the time-dependent nature of the geomagnetic field is well known. Changes occur across a wide range of timescales, from seconds - caused by interactions between the solar wind and the magnetosphere, to several tens of million years - the longest observed timespan between polarity reversals. Here, we focus our attention on the origin of the short (annual to centennial) timescale changes in the geomagnetic field often referred to as secular variation (SV). These changes are a reflection of rapid dynamics taking place in the Earth's core. In this review we describe the current state of theoretical understanding of short timescale core dynamics and the constraints provided by geomagnetic and geodetic observations.

Time-dependent models of the magnetic field at the core-mantle boundary (CMB) (see Gillet et al. 2009a, this issue), computed directly from surface observations (see Matzka et al. 2010, this issue) provide the main source of information for studying the short timescale core dynamics. Of the various aspects of SV, the westward drift is arguably its most well-known and documented feature, with observations dating back more than three centuries (Halley 1683, 1692). Though westward drift appears to be a global phenonemon at the Earth's surface, when one traces it back to its origin at the $\mathrm{CMB}$, it is found to consist primarily of the westward motion of a series of intense field features focused around low latitudes under the Atlantic hemisphere (Finlay and Jackson 2003). Many other components of the secular variation are also now well documented, including geomagnetic jerks (see Mandea et al. 2010, this issue), the rapid growth of the South Atlantic low field anomaly (Gubbins 1987), and anticyclonic motions of field features in the northern hemisphere polar region (Olson and Aurnou 1999).

Though all these processes have been recognized for at least a decade or longer, detailed understanding based on mechanisms of core magnetohydrodynamics (MHD) remain elusive. The westward drift is a case in point; we cannot yet state definitively whether it is produced purely by advection due to a westward equatorial flow or whether phase propagation of a MHD wave is also involved. Furthermore, we do not yet have a clear picture of the underlying dynamical balance sustaining such flows or supporting such waves. A primary goal of this review is to present current hypotheses on the core dynamics underlying $\mathrm{SV}$, pointing out both what can be reliably inferred and the difficulties associated with those aspects that are still debated.

Observed changes in Earth's rotation, a proxy for angular momentum variations, can also be used to probe short timescale core dynamics (Jault et al. 1988; Jackson et al. 1993; Mound and Buffett 2003). However, the precise nature and efficacy of the coupling (electromagnetic, topographic or gravitational) with the lower mantle and inner core is not yet precisely understood. As we show in the pages that follow, continuing uncertainty on such matters is a reflection of the fundamental complexity of the forward problem combined with limitations in our observational knowledge, rather than due to lack of interest or effort in the subject.

Core motions are governed by the equations of rotating MHD and in Sect. 2 we briefly review these along with the main approximations that pertain to short timescales. In Sect. 3 we mention recent insights brought to the subject by $3 \mathrm{D}$ self-consistent numerical simulations of rapidly-rotating, convection-driven, MHD dynamos. In Sect. 4, we review core flow models resulting from the inversion of magnetic observations and discuss possible observational evidence for the existence of wave motions and magnetic diffusion in the core. Finally in Sect. 5 we offer a perspective on promising avenues for future progress. 


\section{Theory}

\subsection{Governing Equations}

An understanding of the mechanisms producing the observed diversity of fluctuations in Earth's core-generated magnetic field requires detailed consideration of how motional induction occurs in the outer core. There, liquid iron alloy is undergoing vigorous convection driven by the cooling of the planet and by chemical differentiation at the inner-core boundary; the core motions stretch, twist and advect the Earth's magnetic field pushing it into new configurations, generating electrical currents and dissipating energy. The theoretical framework for describing such processes is magnetohydrodynamics-the marriage of electromagnetism and hydrodynamics.

Magnetohydrodynamics begins with the assumption that the electrically conducting fluid in Earth's core can be well approximated on the macroscopic scales of interest as a continuum. We further suppose that the fluid obeys Newtonian laws of viscosity and that convection can be adequately represented using the Boussinesq approximation. Then, in a frame of reference rotating with the mantle, the conservation of momentum can be stated in the form of a Navier-Stokes equation (for further details see Gubbins and Roberts 1987) as

$$
\rho_{0}\left(\frac{\partial \mathbf{u}}{\partial t}+\mathbf{u} \cdot \nabla \mathbf{u}+2 \boldsymbol{\Omega} \times \mathbf{u}\right)=-\nabla p+\rho^{\prime} \mathbf{g}+\mathbf{J} \times \mathbf{B}+\rho_{0} v \nabla^{2} \mathbf{u},
$$

where $\rho^{\prime}$ is the departure from the hydrostatic density $\rho_{0}, \mathbf{u}$ is the fluid velocity, $\mathbf{B}$ is the magnetic field, $\boldsymbol{\Omega}$ is the Earth's rotation vector, $p$ is the non-hydrostatic part of the pressure modified to include centrifugal effects, $\mathbf{g}$ the acceleration due to gravity, $v$ the kinematic viscosity, and $\mathbf{J}$ the current density.

The buoyancy term $\rho^{\prime} \mathbf{g}$ is the power source for convective flows and is ultimately responsible for driving fluid motions. If the density perturbations are expressed in terms of temperature perturbations ${ }^{1} \Theta$ away from an adiabatic background state, then

$$
\rho^{\prime} \mathbf{g}=\alpha \rho_{0} g_{0} \Theta \mathbf{r},
$$

where $\alpha$ is the coefficient of thermal expansion, $g_{0}=|\mathbf{g}| / r_{c}$ where $r_{c}$ is the radius of the $\mathrm{CMB}$ and $\mathbf{r}$ is the radial vector.

The term $\mathbf{J} \times \mathbf{B}$ on the right-hand side of (1) represents the Lorentz force through which the magnetic field influences the fluid motions. It can usefully be re-written in the form

$$
\mathbf{J} \times \mathbf{B}=\frac{1}{\mu_{0}}(\nabla \times \mathbf{B}) \times \mathbf{B}=\frac{1}{\mu_{0}}\left[-\frac{1}{2} \nabla \mathbf{B}^{2}+(\mathbf{B} \cdot \nabla) \mathbf{B}\right],
$$

where $\mu_{0}$ is the magnetic permeability. The first term can be considered as a magnetic pressure force (it can be absorbed with the mechanical pressure into a total pressure term) and the second term is associated with tension in the magnetic field lines (see Davidson 2001 for a more detailed discussion).

The evolution of the magnetic field due to the effects of magneto-advection and magnetic diffusion is specified by the magnetic induction equation

$$
\frac{\partial \mathbf{B}}{\partial t}=\nabla \times(\mathbf{u} \times \mathbf{B})+\eta \nabla^{2} \mathbf{B}
$$

\footnotetext{
${ }^{1}$ For a more general formulation of the convection problem in terms of co-density see Braginsky and Roberts (1995).
} 
Table 1 Typical parameter values for Earth's core. References: $1=($ Dziewonski and Anderson 1981), $2=$ (Stacey 2007)

\begin{tabular}{llll}
\hline Parameter & Symbol & Value & Reference \\
\hline Outer core radius & $r_{c}$ & $3485 \mathrm{~km}$ & 1 \\
Inner core radius & $r_{i}$ & $1225 \mathrm{~km}$ & 1 \\
Mean outer core density & $\rho_{0}$ & $1.1 \times 10^{4} \mathrm{~kg} \mathrm{~m}^{-3}$ & 1 \\
Gravitational acceleration at CMB & $|\mathbf{g}|$ & $10.68 \mathrm{~m} \mathrm{~s}^{-2}$ & 1 \\
Gravitational acceleration factor & $g_{0}=\frac{\|\mathbf{g}\|}{r_{c}}$ & $3.07 \times 10^{-6} \mathrm{~s}^{-2}$ & 2 \\
Rotation rate & $\Omega$ & $7.292 \times 10^{-5} \mathrm{~s}^{-1}$ & 2 \\
Kinematic viscosity & $v$ & $10^{-6} \mathrm{~m}^{2} \mathrm{~s}^{-1}$ & 2 \\
Thermal expansivity & $\alpha$ & $10^{-5} \mathrm{~K}^{-1}$ & $4 \pi \times 10^{-7} \mathrm{~kg} \mathrm{~m} \mathrm{~A}^{-2} \mathrm{~s}^{-2}$ \\
Magnetic permeability & $\mu_{0}$ & $4.7 \times 10^{5} \mathrm{~S} \mathrm{~m}^{-1}$ & 2 \\
Electrical conductivity & $\sigma$ & $1.69 \mathrm{~m}^{2} \mathrm{~s}^{-1}$ \\
Magnetic diffusivity & $\eta=\frac{1}{\sigma \mu_{0}}$ & $1200 \mathrm{~K}^{-1}$ \\
Adiabatic temperature difference & $\Delta T$ & $\sim 10^{-15}$ \\
Ekman number & $E=\frac{v}{r_{c}^{2} \Omega}$ & $\sim 10^{-9}$ \\
Rossby (or Magnetic Ekman) number & $R_{O}=\frac{\eta}{r_{c}^{2} \Omega}$ & $\sim 10^{9}$ \\
Rayleigh number & $R_{a}=\frac{\alpha \Delta g_{0} r_{c}^{2}}{\Omega \eta}$ & & \\
\hline
\end{tabular}

where $\eta=1 /\left(\mu_{0} \sigma\right)$ is the magnetic diffusivity and $\sigma$ the electrical conductivity of the core fluid. This equation follows from Maxwell's equations of electrodynamics and Ohm's law applied to moving conductors, under the 'magnetohydrodynamic approximation' where fluid motions are assumed much slower than the speed of light.

Some of the parameters that enter (1-4) are well known for Earth's core. For instance $\rho_{0}$ and $g_{0}$ can be extracted from seismic models of the Earth's interior (e.g. Dziewonski and Anderson 1981). Others, such as v, are not well known, mainly because laboratory measurements cannot be made at pressures and temperatures that pertain to the Earth's core. We present in Table 1 the numerical values of the parameters used in this review.

\subsection{Magnetic Diffusion and the Frozen Flux Assumption}

The term $\eta \nabla^{2} \mathbf{B}$ in (4) represents the effect of magnetic diffusion on magnetic field evolution. It is associated with a characteristic timescale $\tau_{\eta} \sim L_{B}^{2} / \eta \sim 19,000$ yrs for a length scale $L_{B} \sim 1000 \mathrm{~km}$ and a magnetic diffusivity of $\eta=1.69 \mathrm{~m}^{2} \mathrm{~s}^{-1}$ thought to be appropriate for Earth's core. A more rigorous analysis involves setting $\mathbf{u}=\mathbf{0}$ in (4) and solving for the diffusive modes. In the case of a spherical conductor of radius $r_{c}=3485 \mathrm{~km}$ surrounded by an insulator, the slowest diffusive mode is also the one with the largest spatial scale; it exponentially decreases with a characteristic time scale $r_{c}^{2} /\left(\pi^{2} \eta\right) \sim 23,000$ yrs (for more details, see Gubbins and Roberts 1987). Since the timescale associated with magnetic diffusion of large scale field structures is so much longer than the years to centuries of interest for SV, some models of SV simply neglect magnetic diffusion (Roberts and Scott 1965; Backus 1968). This procedure is commonly referred to as the frozen-flux (FF) assumption, because it corresponds to a situation where magnetic field lines are 'frozen' into the core fluid, i.e., they move along with the fluid (Davidson 2001, see, for example).

A simple scale analysis of (4) also suggests that motional induction should dominate magnetic diffusion for large scale magnetic structures. Taking the ratio of the two terms 
on the right hand side of (4), we obtain a useful non-dimensional quantity known as the magnetic Reynolds number

$$
R_{m}=\frac{|\nabla \times(\mathbf{u} \times \mathbf{B})|}{\left|\eta \nabla^{2} \mathbf{B}\right|} \sim \frac{\mathcal{U} L}{\eta},
$$

where $\mathcal{U}$ is the typical magnitude of the flow and $L$ is a typical length scale. Again taking $L \sim 1000 \mathrm{~km}$ and $\eta=1.69 \mathrm{~m}^{2} \mathrm{~s}^{-1}$ and further assuming that $\mathcal{U} \sim 5 \times 10^{-4} \mathrm{~m} \mathrm{~s}^{-1}(16 \mathrm{~km} / \mathrm{yr})$, as inferred from inversions for the large scale flow-see Sect. 4 and Bloxham and Jackson (1991), leads to the estimate $R_{m} \sim 300$. Note however that this analysis involves the major assumption that a single lengthscale $L$ characterizes the dominant structures in both the magnetic field and flow-for important critiques of this viewpoint see Love (1999) and Takahashi and Matsushima (2005). Furthermore only the large scale magnetic field and flow have been considered and boundary layers due to the confining geometry, where magnetic diffusion may become important (Bloxham 1986; Gubbins 1996), have been ignored.

For a more rigorous analysis of the importance of magnetic diffusion to SV we must think about the boundary layers present close to the CMB. If one neglects the effect of mantle conductivity (Benton and Whaler 1983), the main magnetic field and its SV observed at the Earth's surface can easily be downward continued to the CMB. Its radial component can further be continued to the internal side of the CMB, where it interacts with the core flow. The flows in question cannot be those directly at the CMB: there, the flow must vanish to obey a no-slip condition. Infact, two boundary layers develop at the CMB: one is dynamical (it involves viscosity as well as magnetic and Coriolis forces) and the second is magnetic (it involves magnetic diffusion).

The dynamical boundary layer is of Ekman-Hartmann type (Dormy et al. 2007). Its characteristic thickness is $\delta_{E} \sim E^{\frac{1}{2}} L_{u} \sim 3 \mathrm{~cm}$, taking $E \sim 10^{-15}$ (see Table 1) along with $L_{u} \sim 1000 \mathrm{~km}$ as a characteristic spatial scale for the large scale flow. The jumps $\left[B_{r}\right]_{E}$, $\left[B_{H}\right]_{E},\left[u_{r}\right]_{E}$ and $\left[u_{H}\right]_{E}$ of the radial and horizontal components of the magnetic field and of the flow through this dynamical boundary layer may be estimated from the solenoidal nature of $\mathbf{u}$ and $\mathbf{B}$ and assuming a balance between the Lorentz, Coriolis and viscous forces within the boundary layer (Hide and Stewartson 1972). This yields

$$
\begin{aligned}
\frac{\left[u_{r}\right]_{E}}{\mathcal{U}} & \sim \frac{\left[B_{r}\right]_{E}}{B} \sim \frac{\delta_{E}}{L_{u}} \sim 3 \times 10^{-8}, \\
\frac{\left[B_{H}\right]_{E}}{B} & \sim \frac{\rho_{0} \mu_{0} \Omega \mathcal{U} \delta_{E}}{B^{2}} \sim 6 \times 10^{-5}, \\
\frac{\left[u_{H}\right]_{E}}{\mathcal{U}} & \sim 1,
\end{aligned}
$$

where, following Bloxham and Jackson (1991), we have taken $B \sim 0.5 \mathrm{mT}$ as a typical estimate of the magnetic field strength at the CMB and again $\mathcal{U} \sim 5 \times 10^{-4} \mathrm{~m} \mathrm{~s}^{-1}$ or $16 \mathrm{~km} / \mathrm{yr}$ as an estimate of the magnitude of the large scale flow. Both jumps in the magnetic field components are negligibly small and therefore the magnetic field at the CMB may safely be downward continued through to the bottom of the dynamical boundary layer. The same applies to the radial component of the flow but not to the horizontal flow, which jumps from a no-slip zero value to the free stream velocity at the base of the dynamical boundary.

The magnetic diffusion boundary layer owes its existence to a toroidal field within the bulk of the core. As a toroidal field is not a potential field, under our assumption of an insulating mantle it must vanish at the CMB. This condition is obeyed via a skin effect within 
a diffusive magnetic boundary layer of thickness $\delta_{B} \sim\left(\eta T_{B}\right)^{\frac{1}{2}} \sim 23 \mathrm{~km}$, if $T_{B} \sim 10 \mathrm{yrs}$ is the characteristic time scale of the SV (Jault and Le Mouël 1991). The associated jumps of the magnetic field and radial flow velocity are

$$
\begin{aligned}
\frac{\left[u_{r}\right]_{B}}{\mathcal{U}} & \sim \frac{\left[B_{r}\right]_{B}}{B} \sim \frac{\delta_{B}}{L_{B}} \sim 2.3 \times 10^{-2}, \\
\frac{\left[B_{H}\right]_{B}}{B} & \sim 1,
\end{aligned}
$$

where we have again used $L_{B} \sim 1000 \mathrm{~km}$ for the characteristic lengthscale of the large scale field. The conclusion from this analysis is that the horizontal component of the magnetic field is affected by the diffusive processes within this magnetic boundary layer while the FF assumption continues to hold for the radial component. It can therefore be used to connect SV to flow at the top of the core. Moreover, as pointed out by Jault and Le Mouël (1991), the dynamical effect of the magnetic field within the diffusive boundary layer is small, i.e, core flows are not affected by the Lorentz force produced in this magnetic boundary layer.

It is therefore possible to write the radial component of the induction equation (4) just below the Ekman-Hartmann and magnetic diffusion boundary layers (a region hereafter referred to as the 'core surface') in the form

$$
\frac{\partial B_{r}}{\partial t}=-\nabla_{H} \cdot\left(\mathbf{u} B_{r}\right)
$$

where $\nabla_{H}=\nabla-\mathbf{n} \partial_{r}, \mathbf{n}$ being the unit radial outward vector. Note that in deriving this the assumption that $u_{r}=0$ at the core surface has be employed. Equation (11) was first obtained by Roberts and Scott (1965) and has been the main tool used to reconstruct core surface flows from downward continued SV (see Sect. 4).

Although widely used, the FF assumption has often been challenged on theoretical grounds. Magnetic diffusion is expected to become a significant source of secular variation if the flow is varying too slowly, as in case of nearly steady core flows (Gubbins and Kelly 1996; Love 1999; Maus 2008), or if the spatial scale of the magnetic field becomes too small and large magnetic field gradients are present, for example in regions where upwelling flows drive expulsion of toroidal magnetic field from the core into the weakly conducting mantle (Allan and Bullard 1966; Bloxham 1986; Drew 1993; Gubbins 1996, 2007). Recent numerical simulations of the geodynamo, although not yet operating in an Earth-like parameter regime, do show some significant amounts of magnetic diffusion in some locations at the core surface (Amit and Christensen 2008). Such simulations also provide valuable insight into physical processes that can produce field evolution through magnetic diffusion at the core surface-see Sect. 3 for further discussion of this issue.

On the observational side, numerous studies have been devoted to testing the validity of the FF assumption (see Sect. 4.1). The mathematical tools for such tests were derived by Backus (1968), who showed that the necessary and sufficient conditions for the time varying core surface field to be compatible with the FF assumption may be expressed as:

$$
\begin{aligned}
\frac{d}{d t} \int_{S_{0}} B_{r} d S & =0, \\
\left(\frac{\partial B_{r}}{\partial t}\right)_{C} & =0,
\end{aligned}
$$


where $S_{0}$ are all patches delimited by $B_{r}=0$ curves at the core surface and $C$ are all critical points, i.e., intersections of $B_{r}=0$ curves. If these conditions are satisfied, then (11) has at least one solution $\mathbf{u}_{0}$. Otherwise, the time variation of the radial flux through $S_{0}$ patches is entirely caused by magnetic diffusion (Gubbins 1996):

$$
\frac{d}{d t} \int_{S_{0}} B_{r} d S=\int_{S_{0}} \eta \nabla^{2} B_{r} d S .
$$

A weaker (i.e., necessary but not sufficient) set of constraints applies to the unsigned magnetic flux:

$$
\frac{d}{d t} \int_{S}\left|B_{r}\right| d S=0
$$

where $S$ is the entire core surface. This latter constraint is less sensitive to the geometry of the $B_{r}=0$ curves and is thus expected to provide more robust results from observations. Attempts to test such constraints using geomagnetic observations are described later in Sect. 4.1.

\subsection{Magnetostrophic Balance}

Returning to the Navier-Stokes equation (1), we note it may be written in non-dimensional form as

$$
R_{o}\left(\frac{\partial \mathbf{u}}{\partial t}+\mathbf{u} \cdot \nabla \mathbf{u}\right)+2(\hat{\mathbf{z}} \times \mathbf{u})=-\nabla p+R_{a} \Theta \mathbf{r}+\mathbf{J} \times \mathbf{B}+E \nabla^{2} \mathbf{u},
$$

where we have scaled length by the radius of the core $r_{c}$, time by $r_{c}^{2} / \eta$, magnetic field by $\sqrt{\Omega \rho_{0} \mu_{0} \eta}$ and temperature by the adiabatic difference $\Delta T$ from the inner-core boundary (hereafter ICB) to the CMB. With these scalings, geophysically relevant amplitudes of the magnetic field of $2 \mathrm{mT}$ and of the velocity field of $3 \times 10^{-4} \mathrm{~m} \mathrm{~s}^{-1}$ correspond respectively to non-dimensional values of 1 and 1000. The non-dimensional numbers $E=v / \Omega r_{c}^{2}$, $R_{o}=\eta / \Omega r_{c}^{2}$ and $R_{a}=\alpha \Delta T g_{0} r_{c}^{2} / \Omega \eta$ are, respectively, the Ekman number, Rossby number (sometimes called the magnetic Ekman number) and Rayleigh number. Using the values in Table $1, R_{o} \approx 10^{-9}$ and $E \approx 10^{-15}$ while $R_{a} \approx 10^{9}$.

The small $E$ and $R_{o}$ numbers imply that inertial and viscous effects are, to leading order, unimportant, and that the (dimensional) Navier-Stokes equation can be reduced to a state of balance between Coriolis, pressure, buoyancy and Lorentz forces (Taylor 1963)

$$
2 \rho_{0}(\boldsymbol{\Omega} \times \mathbf{u})=-\nabla p+\rho^{\prime} \mathbf{g}+\mathbf{J} \times \mathbf{B} .
$$

This is the so-called magnetostrophic or MAC (Magnetic Archimedes (buoyancy) Coriolis) balance (Moffatt 1978; Jones 2003). It is often considered to be a first approximation to the time-averaged dynamical balance in the Earth's core; departures from this equilibrium are however likely to play an important role, especially in short timescale dynamicssee Sect. 2.6.

\subsection{Tangentially-Geostrophic Core Surface Motions}

Observed temporal changes in the magnetic field are connected to flow at the core surface through (11). However, once Backus' necessary and sufficient conditions are satisfied, there 
remain a large number of possible solutions to (11) and this is one of the main difficulties in recovering meaningful core flows. To alleviate a part of this non-uniqueness, flows can be further constrained to be consistent with dynamical considerations. If core flows obey a magnetostrophic balance, then at the core surface, the horizontal component of (17) reduces to:

$$
2 \Omega \rho_{0} \mathbf{n} \times(\mathbf{u} \cos \theta)=-\nabla_{H} p+\mathbf{L}_{H},
$$

where $\theta$ is the colatitude and $\mathbf{L}_{H}=\mathbf{L}-(\mathbf{n} \cdot \mathbf{L}) \mathbf{n}$ and $\mathbf{L}=\mathbf{J} \times \mathbf{B}$. Neglecting the Lorentz force term $\mathbf{L}_{H}$ in (18) leads to the so-called tangentially-geostrophic (TG) equilibrium, where the horizontal Coriolis force entirely balances the horizontal pressure force (Hills 1979; Le Mouël 1984; Le Mouël et al. 1985):

$$
2 \Omega \rho_{0} \mathbf{n} \times(\mathbf{u} \cos \theta)=-\nabla_{H} p .
$$

The TG equilibrium is a good approximation in some regions of the core surface, but not in others. It necessarily fails on the geographic equator, where $\cos \theta=0$ and therefore the horizontal component of the Coriolis force vanishes (Backus and Le Mouël 1986). Conversely, since we can write $\mathbf{L}_{H}=-\mathbf{n} \times\left(B_{r} \mathbf{J}_{H}\right)$, it is certainly valid along $B_{r}=0$ level curves, provided the mantle is assumed electrically insulating.

Following Chulliat and Hulot (2000), we introduce the ratio of the horizontal Lorentz force to the horizontal Coriolis force at the core surface:

$$
E_{G}=\frac{\left|\mathbf{L}_{H}\right|}{\left|2 \Omega \rho_{0} \mathbf{n} \times \mathbf{u} \cos \theta\right|} \sim \frac{|\zeta|}{\zeta_{c}},
$$

where $\zeta=B_{r} / \cos \theta$ and $\zeta_{c} \sim 2 \Omega \rho_{0} \mathcal{U} /\left|\mathbf{J}_{H}\right|$. At the top of the core, the main contribution to $\left|\mathbf{J}_{H}\right|$ comes from the toroidal field $\mathbf{B}_{T}$ and may be expressed as:

$$
\left|\mathbf{J}_{H}\right| \sim \frac{1}{\mu_{0}}\left|\frac{\partial T}{\partial r}\right|,
$$

where $T$ is the toroidal scalar defined by $\mathbf{B}_{T}=\nabla \times(\mathbf{r} T)$. An upper bound of $5 \times 10^{-8} \mathrm{~T} \mathrm{~m}^{-1}$ for the toroidal scalar gradient is provided by length-of-day variations, regardless of the mechanism at the origin of core-mantle coupling, hence $\left|\mathbf{J}_{H}\right| \leq 4 \times 10^{-2} \mathrm{~A} \mathrm{~m}^{-2}$ (Jault and Le Mouël 1991). Taking the values in Table 1, we find $\zeta_{c} \geq 10^{-2} \mathrm{~T}$. This value of $\zeta_{c}$ is large enough to have an extended region at the core surface where $E_{G} \ll 1$, i.e., where the TG assumption holds. As an illustration, we show in dark grey in Fig. 1 the non-geostrophic region defined by $E_{G} \geq 10^{-1}$ in 2005.0, obtained from the CHAOS-2s model (Olsen et al. 2009) truncated at spherical harmonic degree 13.

The TG assumption significantly reduces the number of possible solutions of (11), as was shown by Backus and Le Mouël (1986). Under the combined FF and TG assumptions, the flow at points connected to the geographic equator by an iso- $\zeta$ level curve (in the socalled 'visible domain') becomes uniquely determined by the SV; the flow in the rest of the core surface (in the so-called 'ambiguous domain') has only its component perpendicular to the local iso- $\zeta$ level curve uniquely determined. A full, explicit solution of this problem was obtained by Chulliat (2004). Note that iso- $\zeta$ level curves cross the equator in a limited number of points (eight in 2005, see Fig. 1). The pressure at any point $M$ connected to a point $N$ on the geographic equator by an iso- $\zeta$ curve is also given by the following simple expression

$$
p_{M}=p_{N}+2 \Omega \rho_{0} \int_{N}^{M} \frac{\partial B_{r} / \partial t}{\left|\nabla_{H} \zeta\right|} \boldsymbol{\tau} \cdot d \mathbf{l},
$$




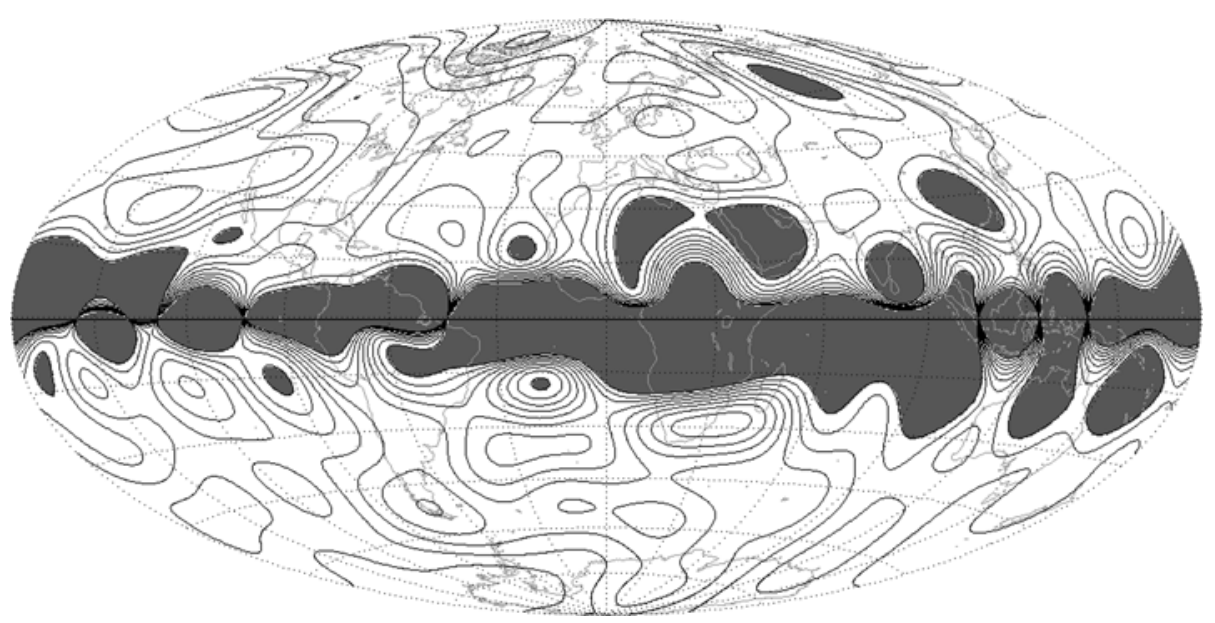

Fig. 1 Non-geostrophic region shown in dark grey at the core surface in 2005.0, defined by $E_{G} \geq 10^{-1}$ or $|\zeta|=\left|B_{r} / \cos \theta\right| \geq 10^{-3} \mathrm{~T}$, see (20), as calculated from the CHAOS-2s (Olsen et al. 2009) geomagnetic field model truncated at spherical harmonic degree 13. $B_{r}$ is the radial magnetic field and $\theta$ the colatitude. Level curves of $\zeta$ are drawn as black, solid lines. Note that contours of $\zeta$ that do not cross the equator delimit ambiguous domains

where $\boldsymbol{\tau}=\mathbf{n} \times \boldsymbol{\pi}$ with $\pi=\nabla_{H} \zeta /\left\|\nabla_{H} \zeta\right\|$, and the integral is taken along the iso- $\zeta$ curve with $d \mathbf{l}$ oriented from $N$ to $M$.

Like for the FF assumption, there is a set of necessary and sufficient conditions on the magnetic field and its SV for the TG assumption to be valid (Chulliat and Hulot 2001; Chulliat 2004):

$$
\begin{aligned}
\oint_{\partial S_{\zeta}} \frac{\partial B_{r} / \partial t}{\left|\nabla_{H} \zeta\right|} \boldsymbol{\tau} \cdot d \mathbf{l}=0, \\
\int_{N_{i}}^{N_{j}} \frac{\partial B_{r} / \partial t}{\left|\nabla_{H} \zeta\right|} \boldsymbol{\tau} \cdot d \mathbf{l}=\frac{1}{2 \Omega \rho_{0}}\left(p_{j}-p_{i}\right),
\end{aligned}
$$

where $\partial S_{\zeta}$ is any closed iso- $\zeta$ level curve, the integral in (24) is taken along any iso- $\zeta$ level curve crossing the geographical equator at $N_{i}$ and $N_{j}$, and $\mathbf{d l}$ is oriented from $N_{i}$ to $N_{j}$. If these conditions are satisfied, then (11) has a unique solution within the visible domain (Chulliat 2004). Generalized forms of these integrals involving diffusion and the Lorentz force were derived by Hulot and Chulliat (2003).

Other integral constraints can be used to test the combined FF $+\mathrm{TG}$ assumption. The vorticity flux through surfaces $S_{\zeta_{0}}$ delimited by closed $\zeta=0$ level curves should remain constant in time (Jackson 1996):

$$
\frac{d}{d t} \oint_{S_{\zeta_{0}}} \cos \theta d S=0
$$

while the magnetic flux through the same surfaces should also remain constant (Backus and Le Mouël 1986):

$$
\frac{d}{d t} \oint_{S_{\zeta_{0}}} B_{r} d S=0
$$


However, these constraints are weaker than (23)-(24) because they are only necessary and not sufficient conditions for (11) to have a solution.

We note that in addition to the TG assumption, other dynamical constraints have been used in conjunction with (11) in order to recover core flows. These include flows specified to be steady (Gubbins 1982), purely toroidal (Whaler 1980), helical (Amit and Olson 2004), or columnar (Amit and Olson 2004). More details on these constraints can be found in the review by Holme (2007). Examples of TG flows derived from SV are presented in Sect. 4.2.

\subsection{Quasi-Geostrophic Core Dynamics}

The TG and FF assumptions discussed in previous sections allow inferences to be made concerning some aspects of the flow at the surface of the outer core. Although useful, this framework provides no link with what is happening in the deeper parts of the core. The dynamical assumption of quasi-geostrophy (QG) couples the core surface flow to the flow throughout the outer core allowing a more complete perspective on short timescale dynamics. In this section we outline the theoretical basis of the QG framework.

In rapidly rotating bodies, because of the strong influence of the Coriolis acceleration, flows often tend to be in a state close to that of geostrophic balance in which the Coriolis force alone equilibrates the pressure force. Purely geostrophic flows possess no variation in the direction parallel to the rotation axis, a constraint known as the Taylor-Proudman ${ }^{2}$ theorem (Proudman 1916; Taylor 1917). In spherical geometry when buoyancy and Lorentz forces are present they naturally force small departures from geostrophy. The resulting motions are referred to as being quasi-geostrophic. Such a regime appears to be appropriate for describing short timescale dynamics in the Earth's core (Jault 2008). Below we present the equations governing QG dynamics and some of their consequences.

We first note that the equation describing a strict (not just tangentially) geostrophic balance is obtained via a further simplification of the magnetostrophic balance (17) by neglecting the final two terms on the right-hand-side and requiring that the Coriolis force alone equilibrates the pressure force,

$$
2 \rho_{0}(\boldsymbol{\Omega} \times \mathbf{u})=-\nabla p .
$$

Taking the curl of (27) yields the Taylor-Proudman theorem,

$$
\frac{\partial \mathbf{u}}{\partial z}=\mathbf{0} .
$$

To remain in exact geostrophic balance, the fluid must therefore move as if rigid along the $z$ direction (along the rotation axis) and fluid parcels must take the form of columns with constant height. This limits the possible geostrophic motions in a spherical shell to co-axial cylinders rotating with velocities $s \omega(s) \hat{\phi}$, where $s$ is cylindrical radius and $\omega(s)$ is the angular velocity. Such flows are particularly important in storing the fluid angular

\footnotetext{
${ }^{2}$ As an historical aside, we note that Sydney Samuel Hough (1870-1922) had earlier derived the same theorem (Gill 1982, p. 506). On p. 208 of Hough (1897) it is noted that for very long period motions $\partial u / \partial z=\partial v / \partial z=\partial w / \partial z=0$ and that 'in the case of tides of very long period the velocity of the fluid particles is approximately the same at all points in the same line parallel to the polar axis while in the case of ocean currents this is rigorously the case'. Nonetheless, since it was the theoretical work by Proudman (1916) following a suggestion by G.I. Taylor (see Proudman 1916; Taylor 1917) and the subsequent elegant experimental work by Taylor $(1921,1923)$ that drew attention to this remarkable property of rotating fluids, thus it is conventionally referred to as the Taylor-Proudman theorem (Chandrasekhar 1961).
} 
momentum and propagating it, for instance via torsional waves (see Sect. 2.6). However, these motions cannot transport heat radially (from inside the core out to the mantle), and are not the solution of any force balance involving buoyancy. Also, they cannot generate and sustain the geodynamo because they are purely planar and hence subject to an anti-dynamo theorem (e.g. Gilbert 2003).

Efforts to understand convecting flows in rapidly-rotating spherical shells by Roberts (1968) and Busse (1970) identified that the relevant modes at the onset of convection, in the asymptotic limit of small Ekman number, consist of non-axisymmetric, equatoriallysymmetric motions involving rolls clustering around the tangent cylinder. For such motions, slight deviations from geostrophy can be treated as a perturbation to a primary geostrophic balance. More recently, Jones et al. (2000) and Dormy et al. (2004) confirmed the relevance of columnar flow solutions by studying the global onset of convection; the columnar convection paradigm has in addition received strong support from both experimental studies (Busse and Carrigan 1976; Carrigan and Busse 1983; Cardin and Olson 1992, 1994) and numerical studies of the full 3D nonlinear governing equations (Tilgner and Busse 1997). Working in cylindrical polar coordinates $(s, \phi, z)$, the fundamental assumption underlying the quasi-geostrophic theory of columnar convection is that, in the limit of high rotation rates, rigidity along $z$ is still present to a large extent so

$$
\frac{1}{s} \frac{\partial}{\partial \phi}, \frac{\partial}{\partial s} \gg \frac{\partial}{\partial z} .
$$

A schematic visualization showing the geometry of QG motions relevant to Earth's core is presented in Fig. 2.

The QG assumption makes it possible to write

$$
\mathbf{u}=\nabla \times[\Psi(s, \phi, z, t) \hat{\mathbf{z}}]+u_{z}(s, \phi, z, t) \hat{\mathbf{z}} .
$$

Fig. 2 Illustration of the geometry of the quasi-geostrophic (QG) motions. Motions are assumed to be equatorially symmetric and invariant parallel to the rotation axis, so they can be extrapolated from the core surface down to equatorial plane where the dynamics are visualized and modelled. $r_{i}$ is the radius of the inner core, $r_{c}$ is the radius of the $\mathrm{CMB}, \theta_{0}$ is the co-latitude of the rim of the TC at the CMB and $\Psi$ refers to the stream function as defined in (30)

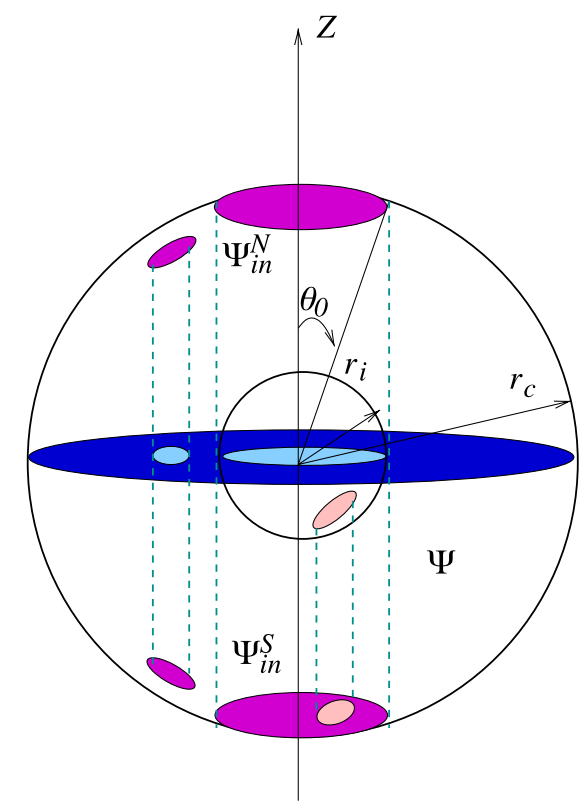


Taking the curl of (1), one obtains an equation governing the fluid vorticity $\xi=\nabla \times \mathbf{u}$. Projecting along $z$ gives a dynamical equation for the evolution of the axial component of vorticity $\xi=\hat{\mathbf{z}} \cdot \xi$. Curling the fluid momentum equation a second time and projecting along $z$ gives an equation which shows that the terms contributing to the $z$-dependence of $\xi$ are all very small (see Jones 2007; Gillet and Jones 2006). The QG approximation consists of neglecting those terms and assuming that the vorticity of the flow is predominantly axial and $z$-independent, i.e., $\partial \xi / \partial z=0$. This reduces the full three-dimensional flow field to

$$
\mathbf{u}=\nabla \times[\Psi(s, \phi, t) \hat{\mathbf{z}}]+u_{z}(s, \phi, z, t) \hat{\mathbf{z}},
$$

where $u_{z} \ll\left(u_{s}, u_{\phi}\right)$. This is in essence a two-dimensional flow since $u_{z}$ can also be written in terms of the streamfunction $\Psi(s, \phi)$ that defines the flow in the equatorial plane

$$
\mathbf{u}_{E}=\nabla \times[\Psi(s, \phi, t) \hat{\mathbf{z}}]=\frac{1}{s} \frac{\partial \Psi}{\partial \phi} \hat{\mathbf{s}}-\frac{\partial \Psi}{\partial s} \hat{\phi}
$$

In the Earth's core, where viscous effects are very small, the $u_{z}$ component is induced mainly by the rigid spherical boundaries, where the non-penetration condition must yield $\mathbf{u} \cdot \mathbf{n}=0$. Then $u_{z}=\mp u_{s} \frac{s}{H_{c}}$ at $z= \pm H_{c}(s)$, with $H_{c}(s)=\sqrt{r_{c}^{2}-s^{2}}$ the (spherical) shape of the rigid core-mantle boundary. Furthermore, fluid mass conservation requires that $u_{z}$ be linear in $z$ (since $u_{s}$ and $u_{\phi}$ are $z$ invariant). These conditions yield

$$
u_{z}=-u_{s} \frac{s z}{H_{c}^{2}}=-\frac{z}{H_{c}^{2}} \frac{\partial \Psi}{\partial \phi}
$$

inside the core, corresponding to $\partial u_{z} / \partial z=-\beta u_{s}$ if we define $\beta=\left(1 / H_{c}\right)\left|d H_{c} / d s\right|$ to be the local slope of the rigid boundary. This $u_{z}$ flow contributes a vortex-stretching term to the dynamical equation for $\xi$ (see (34-35) below). It leads to a $\beta$-effect similar to that in meteorology and oceanography, although the origin of the $\beta$-effect in such constant depth geophysical systems is instead related to the variation of the Coriolis parameter $f=2 \Omega \cos \theta$ with co-latitude. In both kinds of geophysical systems the $\beta$-effect term is responsible for the restoring force at the origin quasi-geostrophic waves commonly referred to as Rossby waves (e.g. Pedlosky 1987).

A central assumption in the QG theory is the requirement that the characteristic lengthscale parallel to the rotation axis be larger than the characteristic lengthscale in the equatorial plane (see (29)). This turns out to require that $\beta$ be kept very small i.e. $\beta \ll 1 / \ell_{e} \Leftrightarrow$ $\left(\sin \theta / \cos ^{2} \theta\right) \ll r_{c} / \ell_{e}$ where $\ell_{e}$ is a characteristic lengthscale in the equatorial plane and $\theta$ is co-latitude. This assumption is no longer true when approaching the equator, where the local surface slope diverges. The QG theory is thus formally invalid at the equator. In addition, we note that a second contribution to the ageostrophic component $u_{z}$, particularly relevant in laboratory and numerical studies, is given by the effect of Ekman suction at the top and bottom of QG columns, typically $E^{1 / 2}$ times the slope-induced circulation (e.g. Aubert et al. 2003; Schaeffer and Cardin 2005).

Let us proceed by formally writing the QG equations governing convective motions, to begin with in the absence of a magnetic field. Taking the curl of the momentum equation and projecting along $z$ we obtain the axial component of the non-linear vorticity equation for rotating convection. When one further neglects viscous effects this may be written as

$$
\frac{\partial \xi}{\partial t}+\left(\mathbf{u}_{E} \cdot \nabla_{E}\right) \xi-(2 \Omega+\xi) \frac{\partial u_{z}}{\partial z}=-g_{0} \alpha \frac{\partial \Theta}{\partial \phi},
$$


where $\nabla_{E}=(\partial / \partial s,(1 / s) \partial / \partial \phi)$. Averaging over $z$, using the $\beta$-effect as the source term for $u_{z}$ at the boundaries, and noting that $u_{s}, u_{\phi}$ and $\xi$ are $z$-invariant gives

$$
\frac{D \xi}{D t}+(2 \Omega+\xi) \beta u_{s}=-\frac{g_{0} \alpha}{2 H_{c}} \frac{\partial}{\partial \phi} \int_{-H_{c}}^{H_{c}} \Theta d z .
$$

This may be interpreted as a dynamical equation for the evolution of the potential vorticity $q=(\xi+2 \Omega) / H_{c}$ moving along with the fluid, in which case it reads

$$
H_{c} \frac{D q}{D t}=-\frac{g_{0} \alpha}{2 H_{c}} \frac{\partial}{\partial \phi} \int_{-H_{c}}^{H_{c}} \Theta d z .
$$

The convective forcing is seen to act as a source term that can change the potential vorticity of the fluid (Aubert et al. 2003).

There is a companion dynamical equation that is necessary to unambiguously define the axisymmetric flow. It is obtained by taking the azimuthal component of the momentum equation and averaging over cylindrical surfaces of radius $s$ and height $2 H_{c}$ :

$$
\frac{\partial \overline{u_{\phi}}}{\partial t}=-\left(\overline{u_{s} \frac{\partial u_{\phi}}{\partial s}}+\frac{\overline{u_{s} u_{\phi}}}{s}\right)
$$

where the overbar denotes an average over $\phi$ and $z$ such as

$$
\overline{f(s)}=\frac{1}{4 \pi H_{c}} \int_{0}^{2 \pi} \int_{-H_{c}}^{H_{c}} f(s, \phi, z) d z d \phi .
$$

This specifies the evolution of the zonal flow $\overline{u_{\phi}}$, in the absence of a magnetic field, when dissipative viscous effects and boundary coupling have been neglected. The driving term is the zonal component of Reynolds stresses. Dissipative terms involving bulk viscous dissipation in the fluid interior and Ekman pumping due to the boundary layer which are responsible for a non-zero $\overline{u_{s}}$ are relevant in laboratory; numerical studies of these aspects have been made by Aubert et al. (2003), Schaeffer and Cardin (2005), Gillet and Jones (2006).

There is no instantly apparent reason for the QG approximation to be valid when one considers the impact of a magnetic field on convection and the back-reaction of the convective flow on the magnetic field. However, a large number of numerical studies of magnetoconvection and convection-driven dynamos in spherical geometry have shown that, provided the fluid is sufficient rapidly rotating, once again a predominantly columnar regime emerges. Convection rolls do however tend to be thicker in the presence of a strong, large-scale, magnetic field (Olson et al. 1999; Jones et al. 2003). At first sight it seems paradoxical that the Coriolis force still constrains the flows to be columnar, even when the Lorentz force attains values of the same order of magnitude. Such comparable force magnitudes are indeed what is expected when approaching the magnetostrophic regime believed to be relevant in the Earth's core. However, as stressed by Jault (2008), the important point is that (provided the inertial terms are not negligible in the fluid dynamical equation) one should compare the relative frequencies of inertial and Alfvén waves when diagnosing the dynamical regime. Jault's argument applies to timescales short compared to the magnetic diffusive time, so seems likely to be relevant for short timescale core dynamics. In this case the appropriate dimensionless number to compare the relative importance of rotational and magnetic effects is the Lehnert number

$$
\lambda=\frac{B}{\Omega \ell_{e} \sqrt{\mu_{0} \rho_{0}}}
$$


which is the ratio of Alfvén and inertial wave frequencies (see (58)). A small $\lambda \sim 10^{-4}$ is anticipated for the Earth's core (Jault 2008, ). Physically, low $\lambda$ corresponds to the component of a disturbance involving shear of flow in the direction parallel to the rotation axis, associated with $(\boldsymbol{\Omega} \cdot \nabla) \mathbf{u}$ in the MC wave equation (56), propagating more rapidly than the component involving shear of the flow along the local magnetic field direction, which is associated with $(\mathbf{B} \cdot \nabla) \mathbf{u}$ in $(56)$.

Assuming that buoyancy forces play a secondary role in QG flow dynamics on short timescales (as may be appropriate for studying short timescale SV as monitored by recent satellite missions) and that the Lorentz forces are instead the main driving mechanism, the appropriate equation governing QG dynamics is (Pais and Jault 2008; Canet et al. 2009),

$$
\frac{d \xi}{d t}+(2 \Omega+\xi) \beta u_{s}=\frac{1}{\rho_{0}} \frac{1}{2 H_{c}} \int_{-H_{c}}^{H_{c}} \hat{\mathbf{z}} \cdot \nabla \times(\mathbf{J} \times \mathbf{B}) d z .
$$

This too can be written in terms of an evolution equation for the potential vorticity $q=$ $(\xi+2 \Omega) / H_{c}$ but with the magnetic field, through the action of the Lorentz force, now acting as a source term

$$
H_{c} \frac{D q}{D t}=\frac{1}{\rho_{0}} \frac{1}{2 H_{c}} \int_{-H_{c}}^{H_{c}} \hat{\mathbf{z}} \cdot \nabla \times(\mathbf{J} \times \mathbf{B}) d z .
$$

In this framework it is the non-linear evolution of the axial component of the curl of the Lorentz force that is responsible for producing changes in potential vorticity and it is thus the crucial ingredient in short timescale core dynamics underlying SV. The companion dynamical equation for $\overline{u_{\phi}}$ in this case is

$$
\frac{\partial \overline{u_{\phi}}}{\partial t}=-\left(\overline{u_{s} \frac{\partial u_{\phi}}{\partial s}}+\frac{\overline{u_{s} u_{\phi}}}{s}\right)+\frac{1}{\rho_{0}} \overline{[\mathbf{J} \times \mathbf{B}]_{\phi}},
$$

which describes zonal accelerations of cylindrical flows, including torsional oscillations, which will be discussed in the next section.

The QG approximation was originally derived in theoretical studies of the linearised vorticity equation relevant for the onset of convection. Nonetheless, thermal and compositional convection in the fully-developed regime, for Rayleigh numbers far above the critical values, have also been shown to exhibit a columnar flow structure (Cardin and Olson 1992, 1994) provided the Ekman and Rossby numbers are kept small. QG models are in fact capable of reproducing many phenomena found in studies of rapidly rotating convecting flows in a sphere (Aubert et al. 2003; Morin and Dormy 2004; Gillet and Jones 2006) and are therefore now beginning to be used in studies of geomagnetic secular variation (Jault 2008; Pais and Jault 2008; Gillet et al. 2009b; Canet et al. 2009). An important advantage in using QG models is that, since they are effectively 2D, they allow the investigation of a range of Ekman numbers (and Lundquist ${ }^{3}$ numbers) unavailable to more complex 3D models with present computing resources, making it possible to approach the conditions of Earth's core. Furthermore, in spite of their simplicity, QG flow also naturally facilitate dynamo action (Olson et al. 1999; Schaeffer and Cardin 2006). Results derived using the QG framework to determine core flows from SV are reported in Sect. 4.3.

\footnotetext{
${ }^{3}$ The Lundquist number $S=L v_{a} / \eta$, where $v_{a}$ is the Alfvén wave velocity (43), describes the ratio of the magnetic diffusion timescale to the timescale of Alfvén waves (see Sect. 2.6). It is expected to be large $\left(\sim 10^{4}\right)$ in Earth's core but this is not the case in present 3D numerical simulations.
} 


\subsection{Torsional Oscillations: Alfvén Waves in Rapidly-Rotating Spherical Geometry}

We have now discussed in some detail various approximations and simplifications of the governing equations commonly made when modelling short timescale core dynamics and the associated magnetic induction. Before turning to the interpretation of geomagnetic and geodetic observations, we finish the theoretical part of this review by highlighting some waves and oscillations supported by the equations of rotating MHD that may be relevant to short timescale core dynamics. We begin by discussing axisymmetric motions known as torsional oscillations. Torsional oscillations are a special example of an Alfvén wave; we start this section by reviewing the physics underlying these most fundamental hydromagnetic waves.

Alfvén waves (Alfvén 1942) are propagating oscillatory motions that can arise in electrically conducting fluids permeated by magnetic fields, provided viscous and magnetic dissipation are sufficiently weak. They result from the competing influence of inertial forces and Lorentz forces. Adopting the FF assumption, a flow directed across magnetic field lines will distort the latter, inducing a Lorentz force opposing further deformation, as specified by Lenz' law. This force acts to reduce the amplitude of the flow, eventually reaching a point where it forces the direction of the flow to reverse. Magnetic field lines are then pushed back to their original, undistorted configuration. Fluid inertia carries fluid parcels past the equilibrium point where there is no Lorentz force and the process then repeats in the opposite direction. In the absence of dissipation this magneto-inertial oscillation leads to transverse waves propagating along field lines that will continue indefinitely. The response of magnetic field lines when perturbed by flow across them is analogous to that of a string when plucked. The Alfvén wave speed, that is the speed at which an Alfvén wave propagates along lines of a magnetic field of strength $B_{o}$, is given by

$$
v_{A}=\frac{B_{o}}{\left(\rho_{0} \mu_{0}\right)^{1 / 2}} .
$$

Thus, the stronger the magnetic field the faster Alfvén waves will travel. Further details including a simple derivation of this result may be found in Davidson (2001).

Given that the Coriolis acceleration is much more important than inertial acceleration for motions in the Earth's core, one might think that Alfvén waves should not be relevant. However, it turns out that Alfvén waves can occur if they involve flows that are not directly affected by the Coriolis force.

Precisely such an arrangement arises if one integrates the $\phi$-component of (17) on cylindrical surfaces aligned with the rotation axis. One can show that such an integral of the Coriolis term must be zero for an incompressible flow. Furthermore, the pressure and buoyancy integrals also vanish identically. Therefore, of the four terms in magnetostrophic balance (17), only the Lorentz integral does not identically vanish and we must have

$$
\iint[(\nabla \times \mathbf{B}) \times \mathbf{B}]_{\phi} s d \phi d z=0
$$

This result is known as Taylor's condition, or Taylor's constraint (Taylor 1963). Solutions that satisfy (44) are said to be in a Taylor state. Further discussion of Taylor states and their historical importance in dynamo theory can be found in the review articles by Fearn (1994, 1998) and Hollerbach (1996). Recent efforts to construct field geometries that obey Taylor's constraint have been made by Livermore et al. (2008, 2009).

In the derivation of Taylor's condition, the inertial and viscous terms were neglected from the outset. Though small, they are expected to make a non-zero contribution to the torque 
Fig. 3 Torsional oscillations: azimuthal oscillations of rigid cylindrical surfaces aligned with the rotation axis. These motions result in Alfvén waves which propagate perpendicular to the cylinders

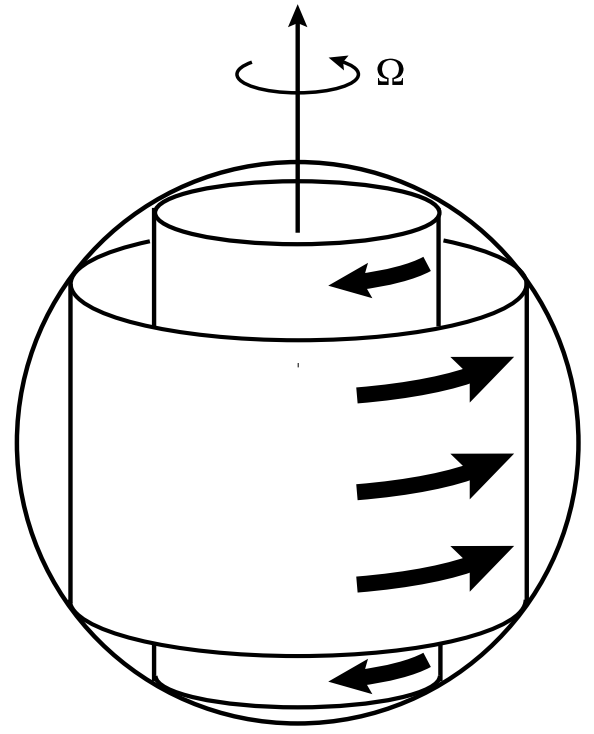

balance on cylinders. The magnetic torque is then not required to cancel exactly but only to be as small as the largest of the neglected torques. With the inertial acceleration term re-introduced, the condition (44) becomes

$$
\left.2 \pi s h \frac{\partial}{\partial t} \overline{u_{\phi}}=\frac{1}{\rho_{0} \mu_{0}} \iint[\nabla \times \mathbf{B}) \times \mathbf{B}\right]_{\phi} s d \phi d z,
$$

where $h=2 H_{c}$ is the height of the cylinder and $\overline{u_{\phi}}$ is the azimuthally averaged velocity of the cylindrical surface. Note that this is an equivalent equation to (42) but without the Reynolds stresses. In the geodynamo literature, $\overline{u_{\phi}}$ is often referred to as the geostrophic flow. Although our scaling analysis of Sect. 2.3 indicated that inertial accelerations should be small, this may not be the case when the timescale of fluid motions $\partial t$ is small compared to the magnetic diffusion time. Lorentz torques in (45) may then be accommodated by 'rigid' accelerations of cylindrical annuli of fluid. The shearing action of the rigid acceleration induces a secondary magnetic field and results in a restoring Lorentz torque. Indeed, coupled with the induction equation, the balance (45) allows oscillations of rigid cylindrical surfaces to propagate about a Taylor state, a situation referred to as a quasi-Taylor state (Braginsky 1970; Dumberry and Bloxham 2003; Takahashi et al. 2005). Such fluid motions are not directly affected by the Coriolis force; the restoring force is purely magnetic. Therefore, these are fundamentally Alfvén waves, though they are commonly referred to in the context of core dynamics as torsional oscillations due to their geometrical similarity to the well known torsional oscillations of solid mechanics. The geometry of torsional oscillations is shown schematically in Fig. 3.

The wave equation for torsional oscillations was first established by Braginsky (1970). In the presence of a 'steady' background magnetic field $B_{s o}, \overline{u_{\phi}}$ must obey

$$
\frac{\partial^{2} \overline{u_{\phi}}}{\partial t^{2}}=\frac{1}{\rho_{0} \mu_{0} s h} \frac{\partial}{\partial s}\left(s^{3} h \overline{B_{s o}^{2}} \frac{\partial}{\partial s} \frac{\overline{u_{\phi}}}{s}\right)-\frac{\partial f_{\phi}}{\partial t},
$$

where $f_{\phi}$ represents the sum of forces that the cylinder exerts on the boundaries, at the ICB and CMB (see Jault 2003 for further details). 
It is important to note that $\overline{B_{s o}^{2}}$ is the average over $\phi$ and $z$ of both the axisymmetric and non-axisymmetric parts of $B_{s o}$. To simplify, let us consider cylinders outside the tangent cylinder (the cylinder tangent to the equator of the inner core) and assume no coupling to the mantle so that $f_{\phi}=0$. We then obtain an equation akin to the equation describing transverse wave propagation on a string

$$
\frac{\partial^{2} \overline{u_{\phi}}}{\partial t^{2}}=\frac{1}{\rho_{0} \mu_{0} s h} \frac{\partial}{\partial s}\left(T(s) \frac{\partial}{\partial s} \frac{\overline{u_{\phi}}}{s}\right),
$$

with $T(s)=s^{3} h \overline{B_{s o}^{2}}$ playing a role similar to tension. The $s$-component of the magnetic field behaves as if it were strings attached to the cylindrical surfaces, providing the restoring force for the waves to propagate in the $s$-direction with Alfvén wave velocity $\sqrt{\overline{B_{s o}^{2}} /\left(\rho_{0} \mu_{0}\right)}$. Note that the azimuthal component of the background field $B_{\phi o}$ does not participate in the restoring force and has no direct influence on torsional oscillations in the absence of magnetic diffusion.

To find the free modes of torsional oscillations, we can substitute into the wave equation (47) normal mode solutions of the form

$$
\overline{u_{\phi}}=U_{o} e^{-i \omega t+i k s} .
$$

The fundamental mode has wavenumber $k=2 \pi / r_{c}$ and its period is $\tau=2 \pi / \omega$, which gives

$$
\tau \approx r_{c}\left(\frac{\rho_{0} \mu_{0}}{\overline{B_{s o}^{2}}}\right)^{1 / 2} .
$$

Using the values in Table 1 and $B_{s o}=0.5 \mathrm{mT}$, we obtain $\tau \approx 25$ years. Exact eigenfunctions of the free modes depend on the spatial variations in $B_{s o}$ and also on the proper incorporation of various coupling effects with the mantle and inner core (Braginsky 1984; Buffett 1998; Jault 2003; Mound and Buffett 2003). Nevertheless, this simple order of magnitude analysis suggests that the normal modes of torsional oscillations should have periods of decades and therefore should participate in the short timescale dynamics of the core. Recent investigations of free torsional oscillations have been performed both in the time (Jault and Légaut 2005) and frequency domain (Mound and Buffett 2003, 2005; Buffett et al. 2009). Note that the decadal time scale inferred above depends crucially on the assumed magnitude of $B_{s o}$ which is not well known. Instead taking $B_{s o}$ to be $2 \mathrm{mT}$, the timescale of the torsional oscillations would be much shorter, on the order of 6 years (Gillet et al. 2010).

\subsection{Magnetic-Coriolis Waves}

In the previous section it was possible to effectively ignore the Coriolis force because we considered azimuthal motions following geostrophic contours for which the pressure gradient exactly balanced the Coriolis force. However, more generally, fluid motions in Earth's core will involve a departure from geostrophy; it is then necessary to consider the combined influence of both the Coriolis force and the Lorentz forces in facilitating wave motions. To understand the consequences for the dynamics of Earth's core we analyze a simplified scenario. We consider an infinite, rapidly-rotating, plane layer permeated by a uniform magnetic field $\mathbf{B}_{\boldsymbol{o}}$ in the absence of viscous and magnetic diffusion. The linearized momentum equation describing the evolution of small perturbations of the flow and magnetic field $(\mathbf{u}, \mathbf{b})$ 
takes the form (see, for example, Davidson 2001, p. 163)

$$
\frac{\partial \mathbf{u}}{\partial t}+2(\boldsymbol{\Omega} \times \mathbf{u})=-\frac{1}{\rho_{0}} \nabla p+\frac{1}{\rho_{0} \mu_{0}}\left(\mathbf{B}_{\mathbf{o}} \cdot \nabla\right) \mathbf{b},
$$

while the frozen flux induction equation simplifies to

$$
\frac{\partial \mathbf{b}}{\partial t}=\left(\mathbf{B}_{o} \cdot \nabla\right) \mathbf{u}
$$

Taking the curl of each of these, using the solenoidal properties of the magnetic and velocity fields, and recognizing the vorticity $\xi=\nabla \times \mathbf{u}$ leads to

$$
\frac{\partial \boldsymbol{\xi}}{\partial t}-2(\boldsymbol{\Omega} \cdot \nabla) \mathbf{u}=\frac{1}{\rho_{0} \mu_{0}}\left(\mathbf{B}_{\boldsymbol{o}} \cdot \nabla\right)(\nabla \times \mathbf{b}),
$$

and

$$
\frac{\partial(\nabla \times \mathbf{b})}{\partial t}=\left(\mathbf{B}_{\boldsymbol{o}} \cdot \nabla\right) \boldsymbol{\xi} .
$$

Taking a further time derivative of (52) and substituting from (53) allows these to be combined into a single equation

$$
\frac{\partial^{2} \boldsymbol{\xi}}{\partial t^{2}}-2(\boldsymbol{\Omega} \cdot \nabla) \frac{\partial \mathbf{u}}{\partial t}=\frac{1}{\rho_{0} \mu_{0}}\left(\mathbf{B}_{\boldsymbol{o}} \cdot \nabla\right)^{2} \boldsymbol{\xi} .
$$

Taking the curl of this and using the property $\nabla \times \xi=\nabla \times(\nabla \times \mathbf{u})=-\nabla^{2} \mathbf{u}$ gives

$$
-2(\boldsymbol{\Omega} \cdot \nabla) \frac{\partial \boldsymbol{\xi}}{\partial t}=\left[\frac{\partial^{2}}{\partial t^{2}}-\frac{1}{\rho_{0} \mu_{0}}\left(\mathbf{B}_{\boldsymbol{o}} \cdot \nabla\right)^{2}\right] \nabla^{2} \mathbf{u} .
$$

Finally collecting terms in $\boldsymbol{\xi}$ in (54) and taking a further time derivative gives an expression for $\partial \xi / \partial t$ that can be substituted back into (55) to eliminate $\xi$ resulting in an equation for the perturbation velocity $\mathbf{u}$ alone. This is known as the 'Magnetic-Coriolis' (MC), 'AlfvénInertial' or 'Magneto-Inertial' wave equation (Lehnert 1954; Acheson and Hide 1973; Davidson 2001),

$$
\left[\frac{\partial^{2}}{\partial t^{2}}-\frac{1}{\rho_{0} \mu_{0}}\left(\boldsymbol{B}_{\boldsymbol{o}} \cdot \nabla\right)^{2}\right]^{2} \nabla^{2} \boldsymbol{u}=-4(\boldsymbol{\Omega} \cdot \nabla)^{2} \frac{\partial^{2} \boldsymbol{u}}{\partial t^{2}} .
$$

We prefer to adopt the terminology 'MC wave equation' for reasons of conciseness and because of its natural extension to the case of MAC waves when Archimedes (buoyancy) forces are also present (Braginsky 1967).

The MC wave equation is considerably more complex than the Alfvén wave equation: it is 4 th order in the time derivative, leading us to expect the existence of 4 different modes. These modes can be isolated by substituting a plane wave ansatz $\boldsymbol{u}=\mathcal{R} e\left\{\widehat{\boldsymbol{u}} e^{i(\boldsymbol{k} \cdot \boldsymbol{r}-\omega t)}\right\}$ into (56) and solving the resulting quartic equation for $\omega$ which yields the following four solutions

$$
\omega_{M C}= \pm \frac{(\boldsymbol{\Omega} \cdot \boldsymbol{k})}{k} \pm\left[\frac{(\boldsymbol{\Omega} \cdot \boldsymbol{k})^{2}}{k^{2}}+\frac{\left(\boldsymbol{B}_{\boldsymbol{o}} \cdot \boldsymbol{k}\right)^{2}}{\rho_{0} \mu_{0}}\right]^{1 / 2}
$$


When the two signs are of the same polarity, then two 'fast' MC waves travelling in opposite directions are obtained. When the two signs are of different polarity, two opposite travelling 'slow' MC waves travelling are obtained.

Note that in the limit where rotation becomes unimportant $(\boldsymbol{\Omega} \rightarrow 0)$ the Alfvén wave dispersion relation $\omega_{M}= \pm\left(\boldsymbol{B}_{\boldsymbol{o}} \cdot \boldsymbol{k}\right) / \sqrt{\rho_{0} \mu_{0}}$ is recovered, while in the limit when magnetic fields are unimportant $\left(\boldsymbol{B}_{\boldsymbol{o}} \rightarrow 0\right)$ the dispersion relation for inertial waves (these are fundamental waves arising from the stability imparted to a fluid by rotation (see Greenspan 1968 , Chap. 4 for details) $\omega_{C}= \pm 2(\boldsymbol{\Omega} \cdot \boldsymbol{k}) / k$ is obtained.

If the frequency $\omega_{C}$ of inertial waves is much larger than the frequency of Alfvén waves $\omega_{M}$ so that

$$
|2(\boldsymbol{\Omega} \cdot \boldsymbol{k}) / k| \gg\left|\left(\boldsymbol{B}_{\boldsymbol{o}} \cdot \boldsymbol{k}\right) / \sqrt{\rho_{0} \mu_{0}}\right|
$$

(i.e. considering large length scales and if rotation is sufficiently rapid; note this criteria is essentially identical to the small Lehnert number criteria $\lambda \ll 1$ proposed for the applicability of quasi-geostrophy, see (39) and Jault 2008) then it is possible to carry out a Taylor series expansion of (57) in the small quantity $k^{2}\left(\boldsymbol{B}_{\boldsymbol{o}} \cdot \boldsymbol{k}\right)^{2} / 4(\boldsymbol{\Omega} \cdot \boldsymbol{k})^{2} \rho_{0} \mu_{0}$. One then finds a very clear splitting of the 'fast' and 'slow' wave frequencies such that the leading order expressions for the dispersion relations are respectively

$$
\omega_{M C}^{f}= \pm \frac{(2 \boldsymbol{\Omega} \cdot \boldsymbol{k})}{k}\left(1+\frac{k^{2}\left(\boldsymbol{B}_{\boldsymbol{o}} \cdot \boldsymbol{k}\right)^{2}}{4(\boldsymbol{\Omega} \cdot \boldsymbol{k})^{2} \rho_{0} \mu_{0}}\right)
$$

and

$$
\omega_{M C}^{s}= \pm \frac{k\left(\boldsymbol{B}_{\boldsymbol{o}} \cdot \boldsymbol{k}\right)^{2}}{2(\boldsymbol{\Omega} \cdot \boldsymbol{k}) \rho_{0} \mu_{0}} .
$$

Remembering that $k^{2}\left(\boldsymbol{B}_{\boldsymbol{o}} \cdot \boldsymbol{k}\right)^{2} / 4(\boldsymbol{\Omega} \cdot \boldsymbol{k})^{2} \rho_{0} \mu_{0}$ is a small quantity, it is observed in this limit that the fast MC wave $\left(\omega_{M C}^{f}\right)$ is essentially an inertial wave slightly modified by the presence of a magnetic field such that the wave frequency is higher than that of a pure inertial wave. Thus frequencies greater than $2 \Omega$ that are impossible for inertial waves are possible for fast MC waves; this can be useful in diagnosing the presence of such waves in experiments (Schmitt et al. 2008).

The slow MC wave $\left(\omega_{M C}^{s}\right)$ that emerges in this rapidly-rotating limit provides a new fundamental timescale

$$
\tau_{M C}^{s} \sim \frac{\Omega \rho_{0} \mu_{0} L_{M C}^{2}}{\pi B_{o}^{2}}
$$

where $L_{M C}=2 \pi / k$ is the lengthscale associated with the slow MC wave disturbance. The period of slow MC waves is decreased with a stronger magnetic field and increased by a faster rotation rate. Physically, slow MC waves are a consequence of a slowly evolving push and pull between the Lorentz force and the Coriolis force; for this reason they are also sometimes referred to as magnetostrophic waves (Moffatt 1978).

The phase and group velocities associated with slow MC waves in the planar geometry in which (60) applies are

$$
\begin{aligned}
C_{\mathrm{ph}} & =\frac{\left(\boldsymbol{B}_{\boldsymbol{o}} \cdot \boldsymbol{k}\right)^{2}}{2(\boldsymbol{\Omega} \cdot \boldsymbol{k}) k \rho_{0} \mu_{0}} \boldsymbol{k}, \\
C_{\mathrm{g}} & =\frac{k\left(\boldsymbol{B}_{\boldsymbol{o}} \cdot \boldsymbol{k}\right)^{2}}{2(\boldsymbol{\Omega} \cdot \boldsymbol{k}) \rho_{0} \mu_{0}}\left(\frac{\boldsymbol{k}}{k}+\frac{2 \boldsymbol{B}_{\boldsymbol{o}}}{\left(\boldsymbol{k} \cdot \boldsymbol{B}_{\boldsymbol{o}}\right)}-\frac{\boldsymbol{\Omega}}{(\boldsymbol{k} \cdot \boldsymbol{\Omega})}\right) .
\end{aligned}
$$


These expressions show that slow MC waves are both anisotropic (their speed depends on the angles between the propagation direction, the magnetic field direction and the direction of the rotation axis) and dispersive (shorter wavelengths have faster phase speeds) while they propagate energy along the magnetic field direction and along the rotation axis as well as normal to the wavefronts (Gubbins and Roberts 1987). It should further be noted that the ratio of the magnetic to kinetic energy for slow MC waves scales like (Acheson and Hide 1973)

$$
\frac{\boldsymbol{b}^{2} / \mu_{0}}{\rho_{0} \boldsymbol{u}^{2}}=\frac{[2(\boldsymbol{\Omega} \cdot \boldsymbol{k}) / k]^{2}}{\left(\boldsymbol{B}_{\boldsymbol{o}} \cdot \boldsymbol{k}\right)^{2} / \mu_{0} \rho_{0}} \sim \lambda^{-2},
$$

where $\lambda$ is again the Lehnert number. Since $\lambda \ll 1$ is a requirement for the splitting into fast and slow MC modes, it may be concluded that for slow MC waves the vast majority of the energy of the disturbance resides in the magnetic field.

An observation of slow (and fast) MC waves was recently reported by Nornberg et al. (2010) in a cylindrical experiment in a rapidly-rotating regime $\left(E \sim 10^{-7}\right)$ with a magnetic field applied coaxial with the rotating axis. This seems to confirm the viewpoint that $\mathrm{MC}$ waves can play a role in natural, rapidly-rotating, magnetic systems even at very high Reynolds number (Nataf and Gagnière 2008).

The slow MC wave may also turn out to be important for understanding SV because $\tau_{M C}^{s} \sim 100-10,000$ yrs for geophysically plausible parameters of $L_{M C} \sim 1000 \mathrm{~km}$ and $B_{o} \sim$ 1-10 mT. It is worth noting that relatively strong fields within the core are required to obtain MC wave timescales comparable to SV. Figure 4 shows the characteristic periods of MC waves (both slow and fast) compared to those associated with torsional oscillations as a function of the underlying magnetic field strength and the typical length scale using (49) and (57). It schematically delineates those free hydromagnetic oscillations that may be of relevance in studies of short timescale core dynamics and SV.

One should however bear in mind that the formulas given in this section and the results collected in Fig. 4 are only crude estimates of the true MC waves timescales in Earth's core since spherical geometry has been ignored, unrealistic uniform fields considered and implausible alignments of field, rotation axis and propagation direction assumed for the sake of simplicity. For example, if there are large cylindrically-radial gradients present in $\boldsymbol{B}_{\boldsymbol{o}}$ then the Lorentz force has an important additional component and it may be possible to obtain $\mathrm{MC}$ waves that are more rapid than suggested by the above analysis.

Some preliminary efforts have been made to compute the properties of MC waves in geometries more appropriate for Earth' s core. Hide (1966) used a $\beta$-plane approach similar to that commonly employed in meteorology and oceanography but modified for a thick spherical shell. He essentially investigated quasi-geostrophic MC waves (or MC Rossby waves) and found these are likely to contribute to SV, though his analysis was limited to very simple uniform imposed fields. Malkus (1967) studied the MC wave problem in the geometry of a full sphere and imposed a simple cylindrically symmetric magnetic field generated by a uniform current density. He was able to obtain analytic solutions for MC modes (also see Zhang et al. 2004) in this special case that were modifications of the classic inertial modes obtained in a rotating sphere (Greenspan 1968; Zhang et al. 2001).

A wide variety of excitation mechanisms exist that could conceivably produce MC waves in Earth's core. These include tidal/precessional forcing (Kerswell 1994), topographic forcing (Hide 1966), shear instability (Fearn 1989), magnetic field instability (Acheson 1972; Fearn 1993) and convective instability (Braginsky 1964; Roberts and Stewartson 1974). In the latter scenario the waves are commonly referred to as MAC waves (where A stands for 


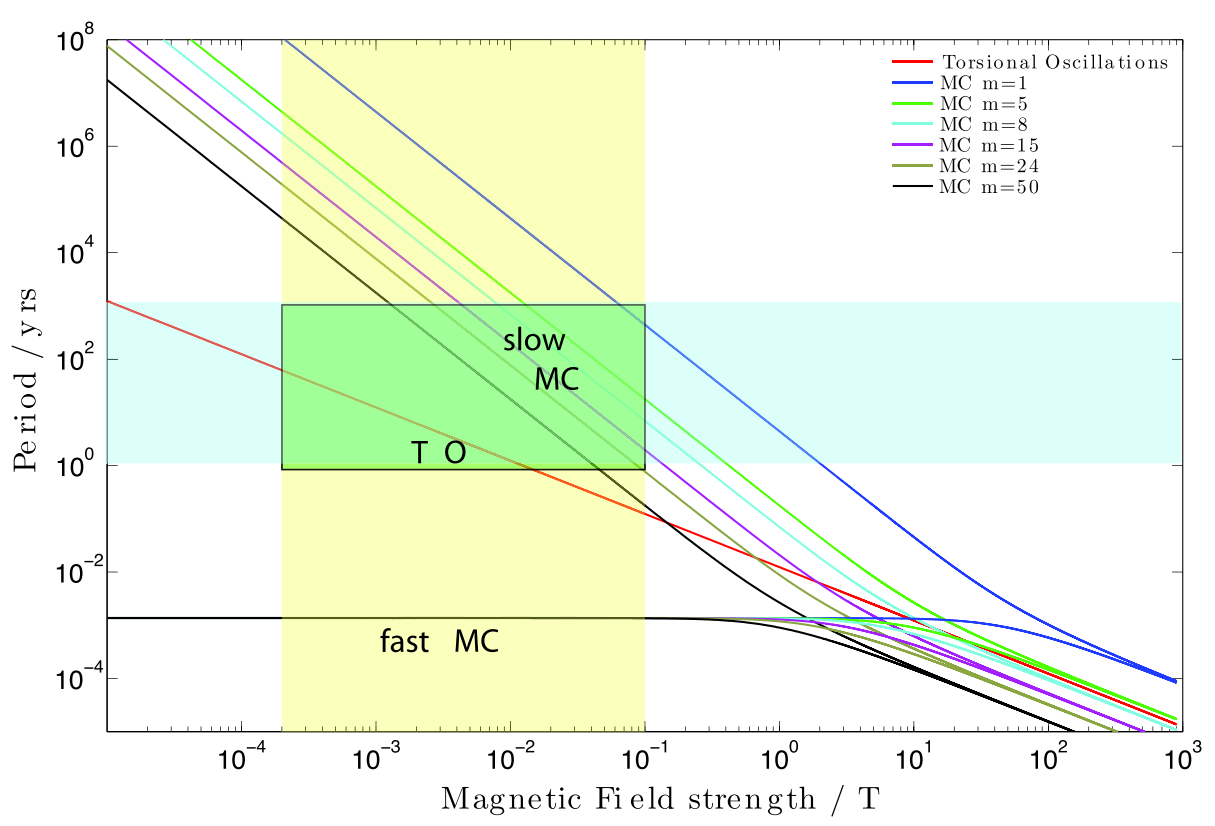

Fig. 4 Periods of MC waves for specified wavenumber $m$, associated with a lengthscale $L_{M C}=2 \pi r_{c} / m$ (dark blue $m=1$, green $m=5$, light blue $m=8$, purple $m=16$, grey $m=24$ and black $m=50$ lines) in comparison with the period of axisymmetric $(m=0)$ torsional oscillations (red line) for a wide range of magnetic field strengths. Values in Table 1 have been used. Note the separation of the MC waves into fast and slow modes as the field strength decreases and rotation begins to dominate magnetic forces. The yellow box highlights a plausible range of magnetic field strengths in Earth's core, the blue box highlights the typical timescales of SV so we suggest that the modes falling within the green box could be relevant for understanding SV. In calculating these curves (57) and (49) have been used and for simplicity it has been assumed that the propagation direction $\boldsymbol{k}$ is parallel to both $\boldsymbol{\Omega}$ and $\boldsymbol{B}_{\boldsymbol{o}}$

the Archimedes driving). MAC waves were first investigated by Braginsky $(1964,1967)$ in a diffusionless framework; later studies demonstrated that diffusion plays a crucial role in the mechanism of their instability (Roberts and Stewartson 1974). We shall not discuss convection-driven MAC waves further in this article because their associated timescales are rather long compared to the annual to centennial timescales of interest here. Readers desiring further details on MAC waves in spherical geometry should consult Proctor (1994), Zhang and Schubert (2000) or Finlay (2008).

\section{Insights from 3D Numerical Simulations of the Geodynamo}

The advent of self-consistent, 3D, numerical models of the geodynamo has in the past ten years revolutionized our understanding of how magnetic field generation might take place in Earth's core (Christensen and Wicht 2007). Such simulations can accurately model the expected kinematics of magnetic induction since they can achieve global magnetic Reynolds numbers of $R_{m} \sim 10^{2}-10^{3}$ (Roberts and Glatzmaier 2000). Current numerical limitations make it challenging to reach a parameter regime appropriate for studying short timescale dynamics that includes, for instance, torsional oscillations and $\mathrm{MC}$ waves resembling those in Earth's core; such regimes are however now beginning to become accessible (Sakuraba 
and Roberts 2009; Wicht and Christensen 2010). Nevertheless, these simulations are a wonderful tool with which to study the details of how nonlinear processes can generate SV in a rapidly rotating, convection-driven, MHD system. They can also be used to explore the assumptions commonly used when modelling short timescale core dynamics, namely FF, TG and QG.

One important issue that has been investigated using dynamo simulations is whether magnetic diffusion (and hence a failure of the FF assumption) is detectable in flux integrals. Roberts and Glatzmaier (2000) used synthetic tests on geodynamo model output to demonstrate that restrictions on spatial resolution severely limit our ability to test the FF and TG assumptions using integral constraints. They compared the differences in unsigned flux (the integral in (15)) between several epochs of their simulation, and were able to distinguish between the influence of magnetic diffusion (FF assumption failure), and the effect of limited resolution due to restrictions on the observable harmonic degree. They concluded that these two sources were of comparable magnitude, hence that magnetic diffusion could not be distinguished from errors due to restricted resolution. In addition, Rau et al. (2000) and Amit et al. (2007) found that the restricted resolution of core field models causes difficulties in the accurate retrieval of core flows by FF-based inversions, particularly if the dynamo is dominated by small scale flows.

Numerical simulations have also been used to investigate in a more general manner the validity of the FF and TG assumptions. Rau et al. (2000) found that the energy of the diffusive contribution to secular variation could reach about $50 \%$ of the advective contribution for a simulation with global $R_{m}=118$, suggesting that the FF assumption may be only a crude first approximation. Their simulation was in better agreement with the TG assumption; the ageostrophic motion represented only a small (less than 10\%) contribution to the total core surface flow. Similarly, Amit and Christensen (2008) found that the relative magnitude of diffusion with respect to the total SV ranged from 0.1 to 0.8 , depending on control parameters of the dynamo simulation. It appears that global $R_{m}$ may be of limited utility in assessing when and where the FF assumption breaks down; the breakdown often occurs locally, for example in small scale vortices associated with intense field concentrations.

An important physical process involving magnetic diffusion that has been studied in numerical simulations (e.g., Takahashi et al. 2005; Aubert et al. 2008) is known as flux expulsion. It involves twisted magnetic fields being pushed out of the core, and occurs when helical convective upwelling flows interact with the toroidal magnetic field close to the core surface. It has been hypothesized that such a flux expulsion event could be the origin of the reversed flux patches currently observed to be growing at the core surface (Gubbins 1987). Since diffusion is difficult to include rigorously in core flow inversions (e.g. Gubbins 1996), one advantage of the numerical simulations is that they allow us to make a connection between flow and magnetic field structures and the process of magnetic diffusion. Amit and Christensen (2008) found that the spatial patterns of radial and tangential diffusion were fairly well correlated. They further identified cyclonic columnar vortices as the physical mechanism at the origin of this correlation and argued that magnetic diffusion may be in balance with advection in several regions of the core surface, for example below the low-latitude Pacific Ocean and northern equatorial Africa.

Some caution is however required when applying results obtained from present numerical dynamo simulations to the Earth. The proportion of diffusion to the total SV in numerical simulations is to a large extent a reflection of their parameter regime. The dominant length scale of the flows and magnetic fields in the low $E$, low $P_{m}$ (where $P_{m}=\kappa / \eta$ is the magnetic Prandtl number), regime of Earth's core is not yet well understood. For example, it is not clear whether the small lengthscale field and flow appearing in many recent simulations 
will continue to dominate or whether larger scales associated with coherent structures will emerge as simulations move to faster rotation rates and to regimes where Ohmic dissipation dominates and stronger toroidal magnetic fields are generated with the omega effect playing a more important role (see, for example, Sakuraba and Roberts 2009). Furthermore, the large Ekman numbers in current simulations also imply thicker and dynamically more important viscous boundary layers. It is unclear to what extend this affects the mechanisms by which they produce secular variation and magnetic diffusion at their outer boundary. The validity of the FF assumption is ultimately related to whether the magnetic field structures are predominantly large scale and whether or not the influence of small-scale flow is negligible. Further study of this issue as Earth-like regimes are approached should be a priority in the years ahead.

Cyclonic and anticyclonic columnar flow vortices are ubiquitous in numerical simulations (see, for example, Olson et al. 1999; Christensen and Aubert 2006; Aubert et al. 2008), thanks to the Taylor-Proudman theorem. This provides strong support for the QG assumption. It has been noted that cyclonic columnar vortices associated with secondary core-surface downwellings can account for the formation at the core surface of stable highlatitude magnetic flux lobes, and short-lived pairs of low-latitude flux features. Extensive investigations of the influence of inhomogeneous thermal boundary conditions on flows in dynamo simulations have also been carried out (see, for example Aubert et al. 2007; Willis et al. 2007 and Aubert et al. 2010 in this issue). A discussion of the origin of features found in flow inversions that draws heavily on this work is given in Sect. 4.2.

\section{Observations and Inversions for Core Flow}

\subsection{Tests of the Frozen Flux Assumption and Evidence of Magnetic Diffusion}

Since the formulation of the FF assumption by Roberts and Scott (1965), significant efforts have been devoted to testing this assumption using geomagnetic observations. Such tests are important because the FF assumption has been widely used to calculate core surface flows, and because a failure would be indicative of magnetic diffusion. The history of the efforts to test the FF assumption has recently been reviewed by Jackson and Finlay (2007). Here we focus on the most recent results.

In principle, direct tests of Backus' condition (12) can be made by simply calculating radial magnetic fluxes through patches delimited by $B_{r}=0$ curves, using a time-dependent geomagnetic field model downward continued to the core surface. Tests of Backus' pointwise condition (13) are even simpler as they only require the knowledge of SV at a particular location. The main difficulty with this approach lies in estimating the error bars on Backus' integral at the core surface, for which several sources of error have to be taken into account: field model errors (due to measurement errors, lack of spatial and/or temporal resolution of the measurements, the influence of regularization (Backus 1988), contamination by external fields), downward continuation errors (due to the weakly conducting mantle) and lack of resolution of the core field due to the masking of its smallest scales by the crustal field.

The availability of high-precision satellite data from the Magsat, Ørsted and CHAMP satellites now makes it possible to test Backus' constraints with greater accuracy than with earlier magnetic field observations. The largest relative change of magnetic flux between 1980 (Magsat data) and the 2000s (Ørsted and CHAMP data) is observed through the St Helena reversed flux patch (Fig. 5, marked by the star), reaching approximately $55 \%$ and corresponding to a total flux change of $50 \mathrm{MWb}$ (Wardinski and Holme 2006; 


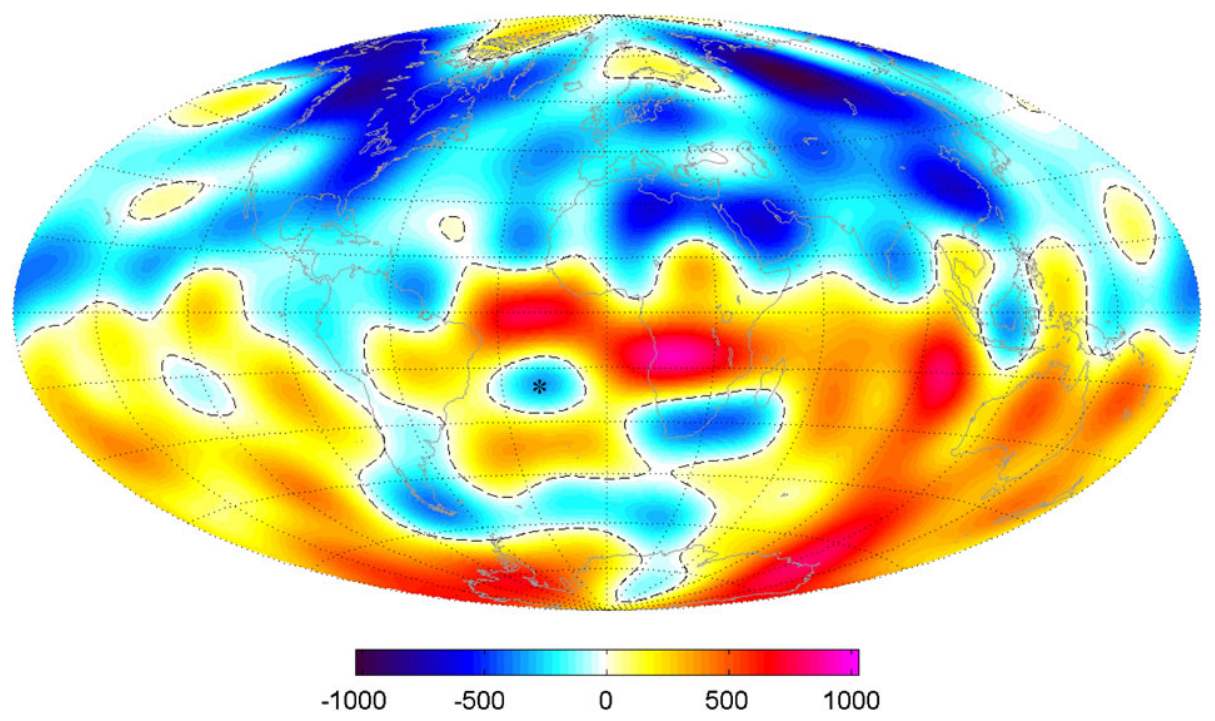

Fig. 5 Map of the radial magnetic field at the core surface from the CHAOS-2s geomagnetic model (truncated at spherical harmonic degree 13), epoch 2005.0. $B_{r}=0$ curves are represented as black dashed lines. St. Helena reversed flux patch is marked by the black star

Chulliat and Olsen 2010). Though the rate of flux change is different, this is consistent with the earlier findings of Bloxham and Gubbins (1986) based on lesser quality observations. A large flux change was also found through the combined South Atlantic/South Pole reversed flux patch by Chulliat and Olsen (2010); however, the relative flux change through this patch is much smaller (5\% in 20 years) due to its very large extent. Another direct observation of magnetic diffusion has recently been reported by Chulliat et al. (2010) in the north polar region of the core surface, below the New Siberian Islands, where a new reversed flux patch seems to have been emerging during the 1990s.

An alternative approach to testing the FF assumption consists of producing core field models constrained to obey Backus' conditions with respect to a reference epoch, and comparing their data residuals with those from unconstrained field models. The conclusion of all such studies (Bloxham and Gubbins 1986; Constable et al. 1993; O'Brien et al. 1997; Jackson et al. 2007; Chulliat and Olsen 2010) is that the FF constraints do not lead to a significant increase in the global misfit ${ }^{4}$ of the model. This indicates that, from an observational perspective, the FF assumption seems to often be justified when studying large scale, short timescale motions. However, this result does not preclude the existence of local failures of the FF assumption, which would only negligibly contribute to the global misfit, either because the amount of magnetic diffusion involved is too small or because Backus' constraints are too weak to effectively constrain core field models. Indeed, Chulliat and Olsen (2010) found that imposing the FF constraints leads to a small but detectable increase of the data residuals at satellite altitude over the St. Helena patch in the 1980-2005 time interval. This result is also consistent with results of direct tests of the FF constraints.

Besides tests of the FF assumption involving Backus' constraints, several recent studies have attempted to estimate the amount of magnetic diffusion at the core surface by other

\footnotetext{
${ }^{4}$ In this context 'global misfit' means the rms difference between the field model predictions and the magnetic observations.
} 
means. Holme and Olsen (2006) calculated the radial SV generated by free decay modes for each spherical harmonic degree of a core field model, which they termed 'static diffusion'. By this technique they estimated diffusive SV would reach about $10 \%$ of the observed secular variation, whatever the degree. In their analysis, the effect of diffusion is maximum at the largest spatial scales, particularly for the axial dipole field. Wardinski et al. (2008) mapped the results of a similar calculation for epoch 2003. Both studies indicated the largest static diffusion would occur within several medium-sized patches at the core surface, including the St Helena patch mentioned above. The relevance of these results to Earth's core where the magnetic field structure is evolving, being driven by advection and not statically decaying is presently unknown; further tests using self-consistent geodynamo simulations may help to shed light on the merits of this approach.

\subsection{Tangentially Geostrophic Core Surface Flow Inversions}

Despite theoretical suggestions and some observational evidence that magnetic diffusion may occur at particular locations at the core surface, the FF assumption has been widely used to invert SV for core surface flows. A detailed review of these efforts is beyond the scope of this paper; see Holme (2007) or Bloxham and Jackson (1991). Here we focus on recent results obtained by inverting (11) for TG flows. As was shown in Sects. 2.4 and 3, the TG assumption is well supported by both theory and numerical simulations over a large fraction of the core surface.

Recent calculations of TG flows rely on several different strategies. The classical method for inverting (11), which involves expanding the flow $\mathbf{u}$ on the TG basis of Backus and Le Mouël (1986) and inverting the resulting linear equation linking SV and flow coefficients, has been used by several authors (Jackson 1997; Pais and Hulot 2000; Hulot et al. 2002; Eymin and Hulot 2005; Asari et al. 2009). Pais et al. (2004) and Holme and Olsen (2006) used a variant of this method, relying on the poloidal/toroidal scalar functions to represent the flow and imposing the TG assumption as an additional constraint by minimizing the rms average of the quantity $\left|\nabla_{H} \cdot(\mathbf{u} \cos \theta)\right|^{2}$ at the core surface. A direct calculation of the TG pressure field in physical space (i.e., not in spherical harmonic spectral space) using (22) was made by Chulliat and Hulot (2000), in an attempt to reduce the impact of the non-uniqueness of the solution within ambiguous patches. Thus far, no direct calculation of the flow using the closed form obtained by Chulliat (2004) has been attempted. Amit and Olson (2004, 2006), Amit et al. (2008) have developed an alternative method relying on the imposition of an additional constraint on the helicity of the flow and carrying out the calculation in the space domain using a grid-based finite difference method. A promising new approach that enables TG flows and core field models to be co-estimated directly from magnetic observations has recently been proposed by Lesur et al. (2010). This has the advantage of requiring just a single inversion whereas traditional methods require two sequential inversions, one to derive a core field model, and a second to derive a flow model.

Below in Fig. 6 from Pais et al. (2004), a flow with TG strongly imposed is shown in (a), a flow with TG weakly imposed is shown in (b) and contours of ageostrophy (defined as $\left.\nabla_{H} \cdot(\mathbf{u} \cos \theta)\right)$ for the latter flow are shown in (c). Note the differences between the flows with strongly and weakly imposed TG, in particular (b) shows some cross equatorial flows that are not found in (a).

The large-scale, zonal, equatorially-symmetric, toroidal part of the reconstructed core flows is, so far, the only part that can be tested against a non-geomagnetic observable. It is now well established that its time variations are correlated with length of day changes that have occurred in the 20th century, which adds further confidence in its derivation from 
(a)
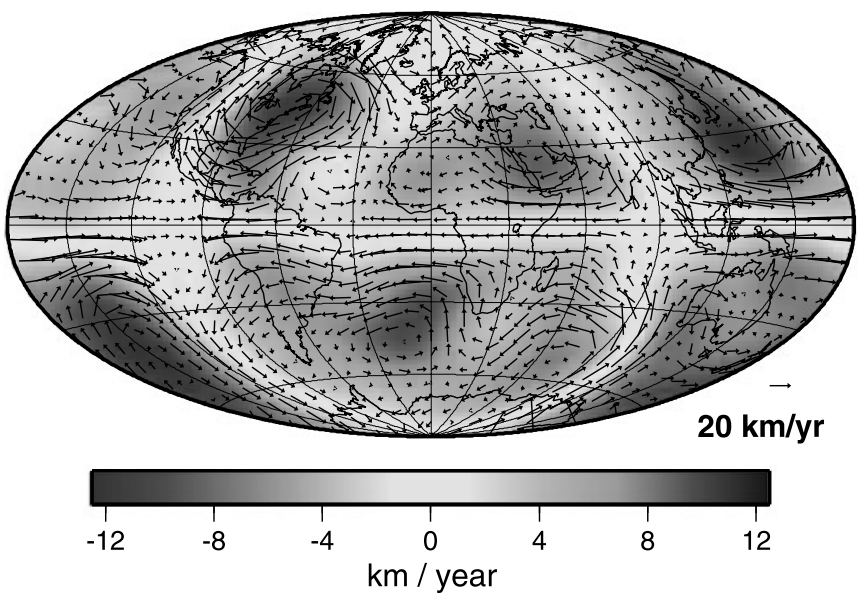

(b)
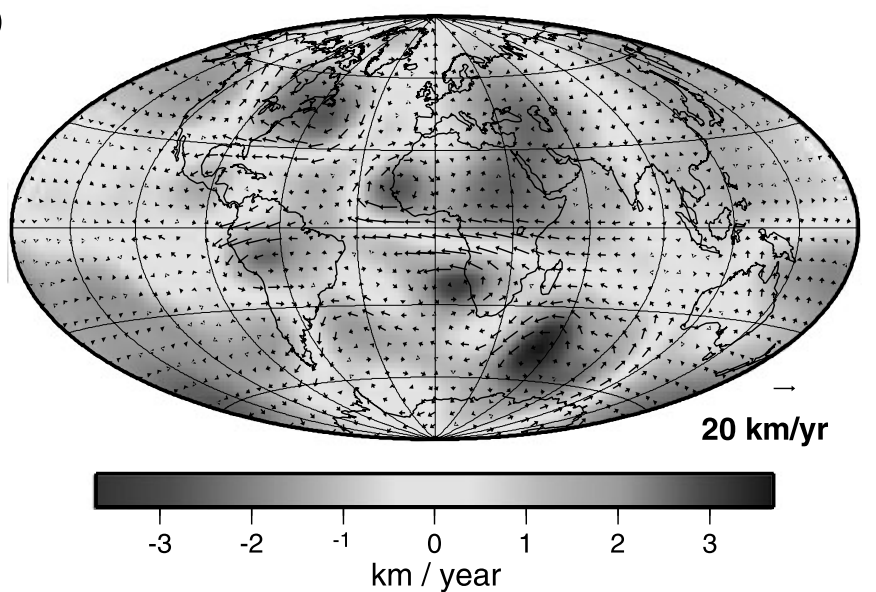

(c)

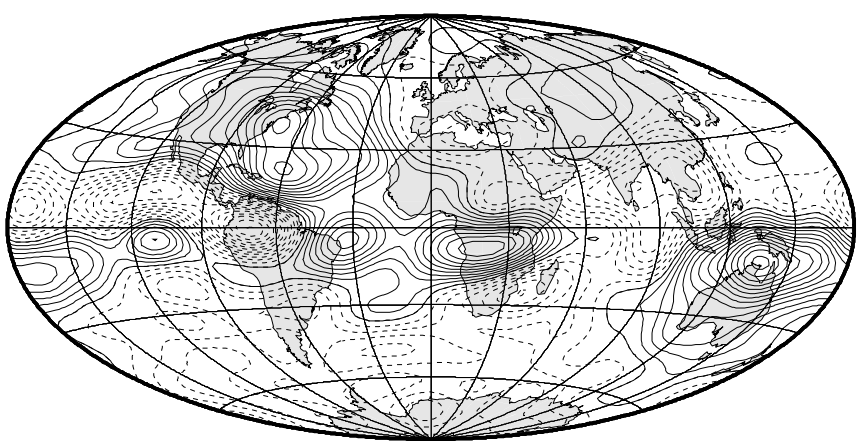

Fig. 6 TG core surface flows from Pais et al. (2004): (a) shows a flow with tangential geostrophy strongly imposed for epoch 1980 (greyscale represents the non-zonal toroidal scalar); (b) shows a weakly tangentially geostrophic flow and (c) shows contours of the ageostrophy, defined as $\nabla_{H} \cdot(\mathbf{u} \cos \theta)$, for the flow in (b) 
geomagnetic data (see Sect. 4.4). Unfortunately, the robustness of other flow features can at present only be tested by comparing flows calculated using different approaches.

When considering the non-axisymmetric part of the flow between 1980 and 2000, the most persistent feature is a giant counterclockwise gyre under the southwestern Indian Ocean. However, its location and size vary significantly from one model to another. In some models, the gyre is centered below the southern tip of Africa, while in others it is located more to the East. Depending on the level of regularization applied to the flow, its size and shape vary from that of a circular gyre spanning about $30^{\circ}$ of latitude (e.g., Hulot et al. 2002) to that of an elongated gyre extending from western Australia to the South Atlantic Ocean (e.g., Holme and Olsen 2006). Another common feature, observed in all TG models cited above, is a clockwise gyre under North America. Again, this gyre is small and circular in some models, being centered under Quebec, while much larger in other models, extending from under the Gulf of Mexico to under Greenland. Other flow features are less robust: the westward flow under the equatorial part of the Atlantic Ocean, observed in many models, is not present in the helical-geostrophic model calculated by Amit and Olson (2006); the flow under the Pacific Ocean, although weak in many models, is quite strong in the model of Asari et al. (2009); westward polar vortices are observed in some models, not all; mid-latitude small-scale vortices, observed by Hulot et al. (2002) using data from the high-precision Ørsted satellite, are absent in more damped models using similar data (e.g., Holme and Olsen 2006).

This very limited number of robust features is a reflection of the many uncertainties involved in the problem of determining core surface flows from geomagnetic data. Sources of errors include: possible failures of the FF and TG assumptions, generation of large scale SV by unresolved small scale flows and non-uniqueness of the TG solution. One could actually question whether the southwestern Indian Ocean and North American gyres are really robust. Both are centered near or within ambiguous domains (see Fig. 1) and are therefore partly extrapolated within such domains by the inversion process. It is certainly possible for small scale flows to generate the large scale SV observed under the southwestern Indian Ocean and North America, thus alleviating the need to have large scale gyres in these areas. This serves as a reminder that large scale non-axisymmetric core flows inferred from geomagnetic data are only one possible interpretation of the SV and should be considered with due caution when discussing core dynamics.

Producing flow maps at the core surface is the first step to establishing a connection between the observed SV and core dynamics. So far rather few studies have attempted to make explicit links between flow features and the aspects interior core dynamics responsible for their existence.

Part of the flow circulation at the surface of the core is believed to be related to the columnar, QG flows inside the core. Given the many arguments supporting QG flows in the core, it is then perhaps surprising that a greater degree of symmetry between the flow structure in the Northern and Southern hemisphere does not emerge from the inversions. It could be argued that the asymmetry reflects the inherent difficulties in core flow inversions and thus flows should be further constrained to obey a QG symmetry from the outset; such inversions are discussed in the next section.

It could however also be argued that imposing equatorial symmetry (as in the QG assumption) fails to account for some flow features at the core surface. In particular, a departure from equatorial symmetry is expected in regions of strong boundary slope at the CMB (at low latitudes) or if there is significant thermal coupling between the core and the bottom of the mantle. Taking $\nabla \times(17)$, using (2) and neglecting the Lorentz force, we recover a 
thermal wind balance (see, for example, Gubbins 1991)

$$
\frac{2 \Omega}{\alpha g_{0} r_{c}} \frac{\partial}{\partial z} \mathbf{u}=\mathbf{n} \times \nabla \Theta
$$

Within the core, temperature perturbations are expected to be small so that the right-hand side of (65) vanishes and we recover the columnar flow structure condition given by (28). This may no longer be true near the CMB; there, large lateral variations in temperature in the lower part of the mantle may imprint lateral temperature gradients at the surface of the core that would affect significantly the structure of flows in a thermal boundary layer near the CMB. Indeed, some of the core flow features shown in Fig. 6 are broadly consistent with a thermal wind pattern derived from (65) when the lateral temperature gradients are based on maps of CMB topography, where lows are assumed to correspond to colder heavier mantle (Kohler and Stevenson 1990; Bloxham and Jackson 1990) or inferred from seismic heterogeneities in the lower mantle (Amit and Olson 2006; Amit et al. 2008).

This scenario has also been tested with numerical dynamo simulations in which lateral variations in heat flow are imposed at the CMB. When the heat flow pattern matches that of lower mantle seismic heterogeneities, core flows near the CMB obey a thermal wind balance and share some broad features with inverted core flows (Olson and Christensen 2002; Christensen and Olson 2003; Aubert et al. 2007). Notably, the Indian-Ocean gyre referred to above is reproduced, with its northern-most limb accounting for a westward drift near the equator under the Atlantic. The North American gyre is also present, but the direction of circulation is reversed. These results suggest that perhaps a portion of the steady, large scale features of core surface flows may indeed be controlled by thermal core-mantle interactions.

Another feature observed in some core flow inversions, the westward polar vortices inside the tangent cylinder (Olson and Aurnou 1999; Hulot et al. 2002), can also be explained in terms of a thermal wind flow. Here, the thermal gradients supporting the flow are not related to the pattern of mantle heat flow but instead to the geometry of convection in the core. Hot buoyant plumes rise from the ICB to the $\mathrm{CMB}$, maintaining the region inside the tangent cylinder at a higher temperature than the region outside (e.g. Glatzmaier and Roberts 1995). This thermal gradient across the tangent cylinder supports a thermal zonal wind, eastward at the ICB and westward at the CMB, in agreement with observations. Numerical simulations also suggest that the strength of Lorentz forces in the convective dynamics is an important ingredient in the formation of strong polar vortices (Sreenivasan and Jones 2005, 2006).

\subsection{Quasi-geostrophic Core Flow Inversions}

QG flows inside the core, on SV timescales, are motivated by the dynamical arguments described in Sect. 2.5. Under the FF and QG assumptions, the core surface radial field $B_{r}$ acts as a passive tracer for the $z$-invariant vortices and these can be reconstructed from geomagnetic field models using standard flow inversion methods (e.g. Holme 2007). QG flows are attractive because unlike TG flows they represent flows inside the core allowing dynamical balances, flow evolution and aspects of the magnetic field within the core to be studied. In fact, QG flows derived by inverting SV can be injected into the dynamical equation (40) governing the short-timescale dynamics inside the core, and the right hand side can be decomposed into various quadratic forms $\left\langle B_{s}^{2}\right\rangle,\left\langle B_{\phi}^{2}\right\rangle$ and $\left\langle B_{s} B_{\phi}\right\rangle$ where averaging has been carried out over the vertical coordinate $z$-see Canet et al. (2009) for details.

In practice, the inversion of (11) for QG flows is implemented by imposing a set of geometrical constraints on solutions: (1) equatorial symmetry outside the tangent cylinder 


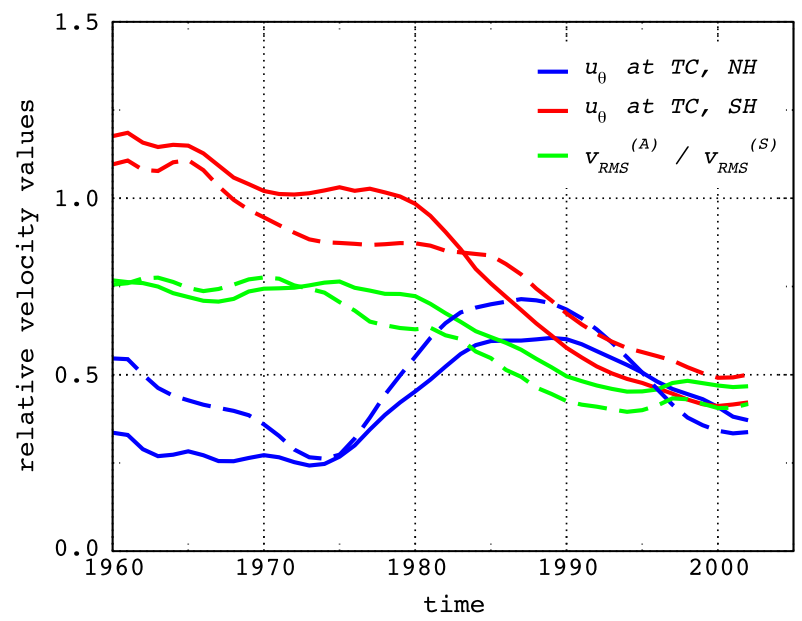

Fig. 7 Relative values of the RMS velocity computed with the equatorially antisymmetric $(A)$ and the equatorially symmetric $(S)$ components of the flow (green curves); the mean longitudinal velocity on the rim of the tangent cylinder divided by the global RMS velocity, both for the Northern (blue curves) and the Southern (red curves) Hemispheres. Dashed lines refer to standard TG flows and solid lines to purely toroidal flows, both computed using the CM4 model (Sabaka et al. 2004) and the $\ell^{3}$ regularization norm of Gillet et al. (2009b), where $\ell$ is the spherical harmonic degree of the flow model. Calculations were made as described in Pais and Jault (2008) with a maximum spherical harmonic degree for the flow representation of 26

(the inner core breaks $z$-invariance inside the tangent cylinder); (2) non-crossing of the tangent cylinder; (3) columnar flow (Pais and Jault 2008; Amit and Olson 2004) or tangential geostrophy, depending on whether or not the volume flow possesses all the required components to assure incompressibility.

Neither equatorial symmetry nor decoupling between the flow inside and outside the tangent cylinder can be unambiguously observed in flow inversions that do not explicitly impose these two constraints (e.g. Amit and Olson 2004). Nevertheless, Fig. 7 shows that the symmetrical component of rms flow $\left(V_{R M S}^{S}\right)$ dominates the anti-symmetrical flow $\left(V_{R M S}^{A}\right)$ during the whole 1960-2002 period. Furthermore, after 1990, not only does $V_{R M S}^{A} / V_{R M S}^{S}$ drop below 0.5 , the flow crossing the rim of the tangent cylinder gets weaker relative to the global $V_{R M S}$ value. The increase in equatorial symmetry during this period seems to follow mainly from a flow reorganization in the South Hemisphere. Thus the two geometrical constraints (1) and (2) do not substantially alter the standard flows obtained by inverting SV. Instead, known features are adjusted in order that these conditions can be exactly verified, particularly for the most recent years when the overall quality of observatory data has improved due to better instruments and the advent of international quality standards (Gillet et al. 2009b; Matzka et al. 2010).

At the core surface, QG flows described by (32) and (33) must respect the same dynamical constraint as TG flows (Pais and Jault 2008), namely

$$
\nabla_{H} \cdot\left(\mathbf{u}_{H} \cos \theta\right)=0 \Longleftrightarrow \nabla_{H} \cdot \mathbf{u}_{H}=\frac{\tan \theta}{r_{c}} u_{\theta}
$$

The well-tested tools of TG inversions can thus be used for QG inversions, including the decomposition of the flow onto a geostrophic basis (Gillet et al. 2009b).

It should however be noted that QG flows of the type defined above are not incompressible. As shown by Amit and Olson (2004), an incompressible QG core flow (or columnar 
flow, as they call it) should satisfy the condition

$$
\nabla_{H} \cdot \mathbf{u}_{H}=2 \frac{\tan \theta}{r_{c}} u_{\theta}
$$

at the core surface instead of (66). Following Schaeffer and Cardin (2005), a possible incompressible QG flow model may be written

$$
\mathbf{u}=\nabla \times\left(\hat{z} \Psi^{*}\right)+\frac{s}{H_{c}^{2}} \Psi^{*} \widehat{\boldsymbol{\phi}}-\frac{z}{H_{c}^{2}} \frac{\partial \Psi^{*}}{\partial \phi} \hat{z},
$$

where $\Psi^{*}$ is a pseudo-stream function.

The first inversions for QG flows were performed only recently (Pais and Jault 2008; Gillet et al. 2009b) using geomagnetic field models constructed from satellite data. These field models have high spatial and temporal resolution and motivated the search for higher resolution inverted flows (Hulot et al. 2002; Holme and Olsen 2006). But, as shown by Eymin and Hulot (2005), small scale flows advecting the unobservable small scale magnetic field contribute strongly to the large scale SV, easily surmounting the observational errors. Thus, inverting for higher resolution flows, irrespective of the underlying dynamical assumption (purely toroidal, TG, QG, columnar or other), requires one to deal with modelling errors resulting from the under-parametrization of the main field. Techniques to tackle this problem include iterative convergence methods (e.g. Pais and Jault 2008) and ensemble methods taking into account the small scale field in a stochastic manner (Gillet et al. 2009b).

Figure 8 shows snapshots for 2001.0 of the QG stream function $\Psi$ in the equatorial plane outside the tangent cylinder, viewed from the North Pole, for QG compressible flow inversions ( $a$ and $b$ ) and a QG columnar (incompressible) flow inversion $(c)$. All three cases are based on xCHAOS field model (Olsen and Mandea 2008) and use the $\ell^{3}$ surface regularization as in Gillet et al. (2009b), where $\ell$ is the spherical harmonic degree of the flow model. For snapshot $a$ the iterative inversion procedure was used, while $b$ represents the robust flow estimate from the ensemble method. All three snapshots have very similar flow features and intensities, including an eccentric (relative to the rotation axis) and planetary scale anticyclonic gyre encircling large to medium scale vortices mainly distributed beneath the Atlantic Hemisphere. One single main cyclonic vortex is responsible for the SV originating beneath the Pacific Hemisphere. The large anticyclonic gyre carries most of the core angular momentum (Pais and Jault 2008) and time fluctuations in its intensity are likely due to some dynamical mechanism involving the coupling to the mantle because, at least during the era when satellite data has been available, they are well compensated by decadal variations in the length of day (Gillet et al. 2009b).

There is a clear correspondence between some core surface features of QG flows and the most common TG flow features described in Sect. 4.2. Because of the imposed equatorial symmetry, however, the most prominent gyres discussed previously gain a symmetrical companion. This is the case for the counterclockwise gyre between 0 and $90 \mathrm{E}$ in longitude, that appears as part of an anticyclone in all three plots in Fig. 8. The western flow under the Atlantic Ocean is also present, equally distributed between the two hemispheres, but has been continued by longitudinal flows to join a high latitude westward flow under the Bering Sea, as previously seen in TG and helical flows (Holme and Olsen 2006; Amit and Olson 2006). This whole feature together forms what is known as the grand eccentric westward jet (Pais and Jault 2008). 

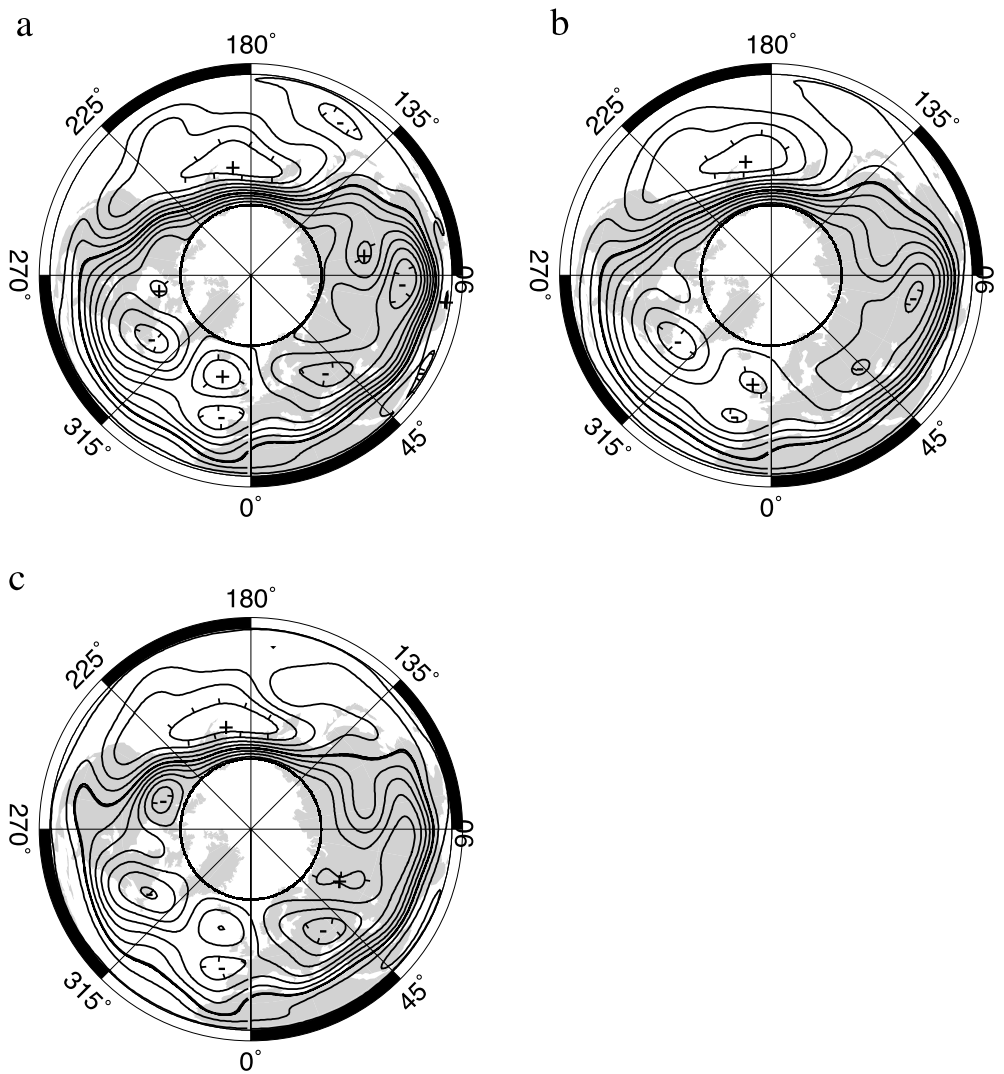

Fig. 8 Stream function $\Psi$ contours (top snapshots (a) and (b)) in the equatorial plane as viewed from the North Pole, for compressible QG flows inverted from xCHAOS-2001.0. Snapshot (a) obtained using the iterative inversion procedure, (b) obtained using the ensemble method. Pseudo-stream function $\Psi^{*}$ contours (bottom snapshot (c)) at the equatorial plane as viewed from the North Pole, for an incompressible QG flow

\subsection{Observations of Torsional Oscillations and Implications for Core-mantle Coupling}

Braginsky (1970) was the first to exploit the theoretical concept of torsional oscillations in an effort to explain geophysical observations. He sought to explain the decade variations in the length of day (LOD) as exchanges of angular momentum between the mantle and the core, with the angular momentum of the core being carried by rigid cylindrical zonal flows.

Braginsky's original idea has since received further support. Jault et al. (1988) have reconstructed maps of the flow at the top of the core between 1969 and 1985 under the assumption of TG. On the basis that the axisymmetric and equatorially symmetric zonal part represents rigid cylindrical zonal flows, they then calculated the changes in core angular momentum and showed that it correlates well with the changes in mantle angular momentum required to explain the LOD variations during that period. Jackson et al. (1993) subsequently showed that this correlation extends back to 1900 . The fit between the observed LOD variations and predictions based on various core flow models is shown in Fig. 9. An example of the time-dependent rigid zonal flows in the Earth's core retrieved from the SV is shown in Fig. 10 for the period between 1900 and 1990. Core flows reconstructed from satellite data suggest that, over the past 10 years, an equally good correlation exists between observed and 
Fig. 9 The observed changes in LOD (black line) versus predictions reconstructed under the assumption that the equatorially symmetric, axisymmetric zonal flows extend rigidly inside the core. The core flow models shown are: purple squares = Jault et al. (1988); blue $=$ Jackson (1997); green $=$ Hide et al. (2000); red = Pais and Hulot (2000)

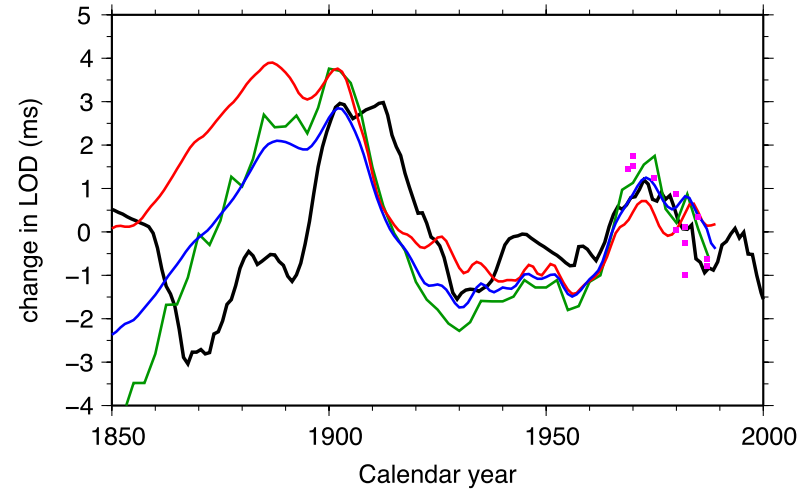

predicted LOD variations (Olsen and Mandea 2008). This supports the idea that changes in LOD at decadal timescales are caused by core-mantle angular momentum exchanges and demonstrates the presence of decadal timescale rigid zonal motions in the core. However, the full story is likely more complicated than this: between 1990 and 2000, core flows predict a much larger change of LOD than is observed (Gillet et al. 2009b). It is unclear at present what is the cause of this discrepancy, for instance whether it can be explained by the presence of non-rigid flows or by a failure to account correctly for unobserved small scale flow when high quality satellite data is not available.

In a quasi-Taylor state context, these rigid flows can be interpreted as the free modes of torsional oscillations propagating about a Taylor state. Indeed, the spatio-temporal variations of the rigid flows (Fig. 10) suggest large exchanges of angular momentum inside the core and relatively little exchange with the mantle (Jault et al. 1996; Zatman and Bloxham 1997; Hide et al. 2000; Pais and Hulot 2000) a feature tending to support the free mode scenario. Furthermore, the decade timescale of the waves is consistent with the range of periods possible for the free modes (given the uncertainty in the field strength within the core), and the observed motions can be explained by a superposition of free waves (Zatman and Bloxham 1997; Bloxham et al. 2002; Amit and Olson 2006).

If this is correct, then torsional oscillations present an opportunity to study physical quantities inside the core or at the CMB for which we otherwise have little or no information. Since the free modes of torsional oscillations must obey (46), the parameters that enter this equation can be retrieved from the observed free oscillations. This idea has already been exploited to extract the possible amplitude and structure of $B_{s}$ inside the core (Zatman and Bloxham 1997), to constrain the coupling mechanism between the core and the mantle that enters through the parameter $f_{\phi}$ (Buffett 1998; Mound and Buffett 2003, 2005), or to do both simultaneously (Buffett et al. 2009).

Although the interpretation of the rigid motions depicted in Fig. 10 in terms of free modes of torsional oscillations may be compelling, it may not be correct. In the free mode scenario, the $B_{s}$ field is chosen or computed with the underlying assumption that the longest periodic features in Fig. 10 correspond to the fundamental mode of torsional oscillations. But this remains an arbitrary choice. For instance, if $B_{s}$ in the core is closer to 1-2 $\mathrm{mT}$ in amplitude, the period of the free modes would be shifted to smaller values. Indeed, a 6-yr oscillation has been observed in the LOD variations (Abarca del Rio et al. 2000), and while this could represent the free mode of gravitational oscillation between the mantle and inner core (Mound and Buffett 2006), it can also be explained by 'fast' torsional oscillations with a fundamental mode of 6 years. The later scenario has been supported by the recent work 


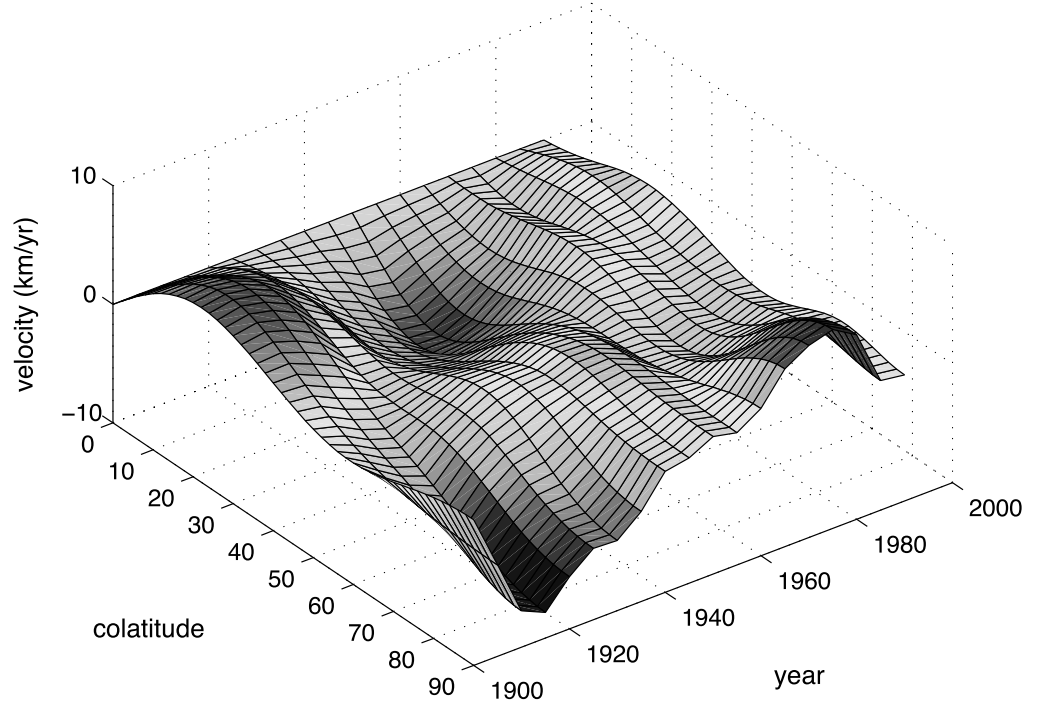

Fig. 10 An example of the time-dependent axisymmetric, equatorially symmetric part of the azimuthal velocity at the surface of the core between 1900 and 1990 inverted from magnetic field model $u$ fm 1 of Bloxham and Jackson (1992). Colatitudes $(\theta)$ are given in degrees. If this part of the velocity extends rigidly inside the core, then it represents time-dependent velocities of rigid cylindrical surfaces with radius $s=r_{c} \sin \theta$. The undulations in radius and time are suggestive of a propagating wave consistent with torsional oscillations

of Gillet et al. (2010) based on the QG framework. It requires that the field in Earth's core be rather stronger $\left(B_{s}>2 \mathrm{mT}\right.$, Canet 2009) than previous estimates had suggested (Zatman and Bloxham 1997; Buffett et al. 2009) in agreement with dynamo scaling laws (Christensen and Aubert 2006) and recent low Ekman number simulations (Sakuraba and Roberts 2009).

If the decadal rigid zonal motions depicted in Fig. 10 are not free modes, they must instead represent geostrophic motions forced by an evolving geodynamo, whether in a Taylor state or otherwise. Even if the fundamental mode of torsional oscillations has a period commensurate with the timescale of variations in Fig. 10, these observed rigid motions could still be dominated by 'forced' rigid motions. This is because over a few decades, free modes are expected to be highly attenuated by coupling with the mantle (Dumberry and Mound 2008) so they need a continuous excitation. The behaviour described in Sect. 2.6, where torsional oscillations occur with respect to a steady equilibrium state, is correct only if they are excited by a mechanism which acts suddenly and then plays no further role in the dynamics. A perhaps more likely scenario is one where cylindrical surfaces are subject to a forcing which plays an active role in the dynamics at all times. If the latter view is correct, the time-dependent rigid flows of Fig. 10 must then include forced oscillations, and the latter may dominate the observed motions.

In principle, numerical simulations of the geodynamo can help discriminate between the free and forced oscillations scenarios. This remains difficult to do at present because at Ekman numbers of $10^{-5}$, typical of current numerical simulations, the viscous torque remains of comparable magnitude to the Lorentz torque with no need for the magnetic field to be self-organized in a Taylor-like manner. Additionally, Reynolds stresses (see (42)) tend to be important in the torque balance (Dumberry and Bloxham 2003), leading to a domination of forced oscillations. However, recent simulations have entered a regime where free torsional oscillations are starting to emerge (Busse and Simitev 2005). In a recent effort focused on 
this specific issue, Wicht and Christensen (2010) have shown that geostrophic flows propagating as free Alfvén waves in the $s$-direction following the dynamics of (47) are indeed observed in their model. The typical wavelength of these Alfvén waves is much smaller than the core-size motion observed in Fig. 10. Nevertheless, the ability of numerical simulations to produce geostrophic flows that can propagate in a manner akin to Alfvén waves is a step in the right direction. As the models are becoming increasingly close to Earth-like conditions, there is hope that they will soon encompass realistic free and forced torsional oscillations.

\subsection{Observational Evidence for Slow Magnetic-Coriolis Waves in Earth's Core}

Observational evidence for the existence of slow MC waves in Earth's core and their relation to aspects of SV were first presented in the studies by Hide (1966) and Braginsky (1972). The advent of modern core surface field models has allowed a more detailed investigation. Using high quality satellite data Jackson (2003) pinpointed the existence of a wavetrain involving a series of maxima and minima of the radial field at low latitudes under the Atlantic hemisphere. Finlay and Jackson (2003) built on the earlier work of Bloxham et al. (1989) and Jackson et al. (2000) and studied the motions of such features in the historical record. They highlighted that the westward motion of the equatorial wave-train was largely responsible for the well known phenomenon of westward drift. A time-longitude plot showing the westward motion of wave-like field features at the equator from the study of Finlay and Jackson (2003) is presented in Fig. 11.

It should be noted that this observation comprises two parts: (i) A series of positive and negative perturbations in the radial magnetic field has existed for at least 400 years at low latitudes at Earth's core surface; and (ii) the pattern has moved westwards in a fairly coherent manner at a speed $\sim 17 \mathrm{~km} / \mathrm{yr}$ during this time. Phase propagation of a slow $\mathrm{MC}$

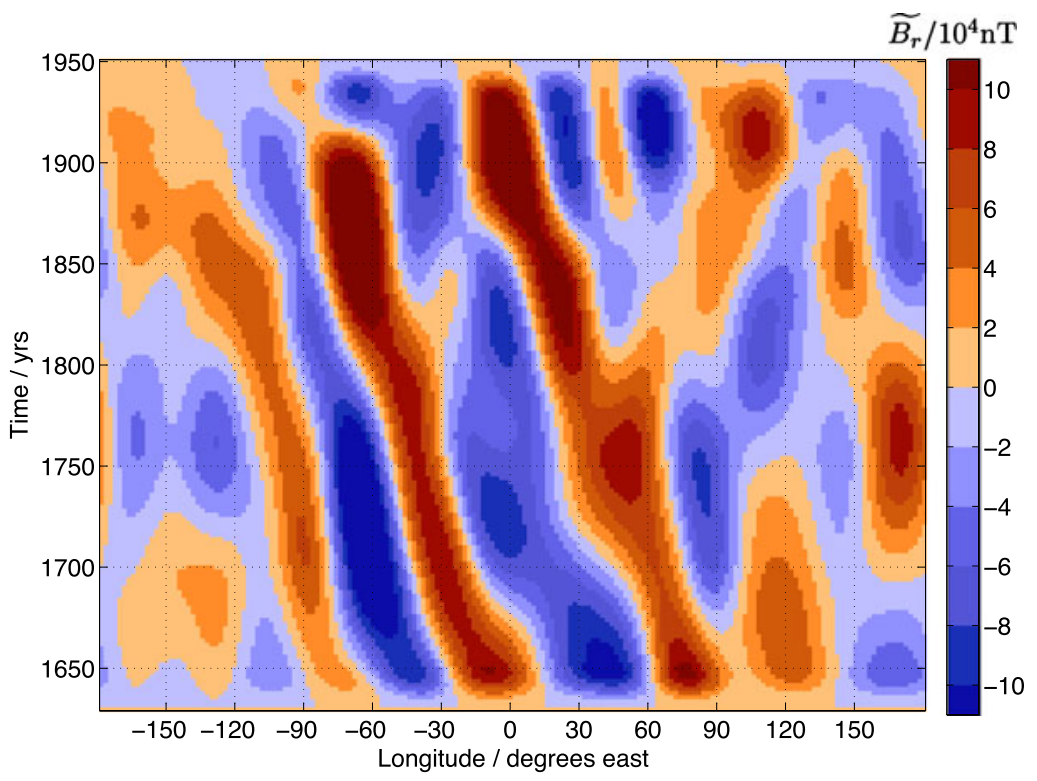

Fig. 11 Time-longitude plot of non-axisymmetric wave-like variations in $B_{r}$. Radial field at the core-mantle boundary over the past four centuries with time-averaged axisymmetric field removed and filtered to retain only variations on timescales shorter than 400 years, adapted from Finlay and Jackson (2003) 
wave along a predominantly azimuthal toroidal field generated by the geodynamo mechanism within the core may be able to simultaneously explain both observations. However it is also possible that an instability could generate slow MC (or MAC) waves with much longer periods (almost stationary on the timescales of interest here) that are subsequently advected westwards by a bulk azimuthal flow. The later scenario is broadly consistent with the structure of the thermal-wind flow expected near the CMB (see Sect. 4.2). Perhaps the most plausible situation is that both slow MC waves and advection by core winds play a role-better forward models of both processes are needed in order to distinguish between these mechanisms using observations.

Interest in MC waves has been revived by recent experimental results (Schmitt et al. 2008; Nornberg et al. 2010) and the availability of numerical solutions for arbitrary axisymmetric imposed fields in spherical geometry (Schmitt 2010). The application of such tools to regimes appropriate for Earth's core should help to clarify whether phase propagation of slow MC waves could play a role in SV. Further forward modelling is also needed to study the role of possible excitation mechanisms (are MC waves in the core free or forced?) and to determine whether slow MC waves would be strongly damped by core-mantle coupling mechanisms.

Unfortunately, precise models of the spatial structure and exact periods of slow MC waves in Earth's core cannot yet be obtained using a forward modelling approach due to our ignorance of the structure and magnitude of the underlying magnetic field within Earth's core. This knowledge is a pre-requisite for calculations of wave properties. Perhaps methods of data assimilation that have already been applied to the torsional oscillation problem (Gillet et al. 2010) will eventually provide a solution to this dilemma.

\section{Concluding Remarks and Outlook}

In this review article we have described recent developments in the science of short timescale core dynamics from the perspective of theory, numerical simulations and observations.

A central issue remains how best to simplify the full governing equations to adequately model the essential fast dynamics of the rapidly-rotating MHD regime present in Earth's core. Significant progress has however been made, particularly with the recent development of the QG framework (Jault 2008; Pais and Jault 2008; Gillet et al. 2009b; Canet et al. 2009). This approach is already yielding some thought provoking results (Gillet et al. 2010) suggesting that the field within the core may be rather stronger ( $>2 \mathrm{mT})$ than previously estimated. Nonetheless, the QG framework is still in its enfancy. Additional work is required, for example to clarify over what range of timescales it holds, since it appears to be incompatible with time-averaged properties of dynamo simulations (Aubert 2005). Furthermore it is at present unclear how to reconcile the QG approach with inhomogeneous CMB thermal boundary conditions (Aubert et al. 2007; Willis et al. 2007; Takahashi et al. 2008b). The consequences of its formal breakdown in the equatorial region should also be explored in the future.

Proposed simplified models of short timescale dynamics should, when possible, be tested against results from self-consistent 3D spherical shell geodynamo simulations in regimes as close as possible to that of Earth's core. Such tests are necessary in order to understand under which circumstances the approximations adopted work well and when they can lead to inaccuracies. Simulations with Ekman number as low as $10^{-5}-10^{-7}$ (Takahashi et al. 2005, 2008a; Kageyama et al. 2008; Miyagoshi et al. 2010; Sakuraba and Roberts 2009; Wicht and Christensen 2010) are now possible. The recent calculations by Sakuraba and 
Roberts (2009) are particularly impressive, producing a convection-driven dynamo with higher magnetic energy than kinetic energy, and with emergent large scale structures in the field and flow in addition to small scale details. Snapshots of the flow in such simulations show a clear dominance of QG motions but also some evidence of diffusive flux expulsion events, particularly at low latitudes.

Observations from recent and upcoming satellite missions are also beginning to stimulate new perspectives on short timescale core dynamics. Field models spanning the satellite era (since 1999) such as the CHAOS series (Olsen et al. 2006, 2009; Olsen and Mandea 2008) and the GRIMM series (Lesur et al. 2008) now provide a globally reliable picture of the core field up to at least spherical harmonic degree 13 as well as detailed information concerning the SV and SA (secular acceleration). Through such monitoring efforts, it has become apparent that the evolution of the geomagnetic field on sub-decadal timescales is punctuated by the rapid secular variation events of duration $\sim 1 \mathrm{yr}$ characterized by geographically localized foci (Olsen and Mandea 2008).

Rapid SV events are certainly produced by some form of short timescale core dynamics but their precise origin and relation to more global geomagnetic jerks is presently unknown. They may be caused by rapid local changes (accelerations) in core flow driven by intense convective fluctuations, or perhaps by rapid fluctuations in the Lorentz force due to reorganization events in the core field (Hide 1985). Another possibility is that they are related to diffusional processes such as a change in the rate of flux expulsion, that may occur on short timescales if very large radial field gradients are present.

As the time interval monitored becomes longer and with the prospect of a constellation of magnetic satellites to be deployed in the ESA SWARM mission (Friis-Christensen et al. 2006), which should allow a more complete picture of external field fluctuations, there is hope that such events can be even more precisely characterized in the years ahead. Unfortunately, it is unlikely that this improved geomagnetic picture can be supplemented by further useful geodetic information, at least in the very near future. Gravity variations obtained by current satellite missions such as GRACE (Tapley et al. 2004) can in principle recover a signal of core origin (e.g. Dumberry 2010). However, this will require the careful removal of interannual gravity variations caused by surface processes which are an order of magnitude larger and mask the core signal.

An ultimate goal of studies of short timescale core dynamics is to understand the physical mechanisms underlying SV well enough to be able to accurately predict future changes in the geomagnetic field, for example up to 5 years ahead. In this scheme inspiration may be drawn from similar quests in oceanography and meteorology where the techniques of data assimilation have recently been developed; for a timely introduction to this subject from the perspective of geomagnetism readers should consult the review article by Fournier et al. (2010) in this issue. Data assimilation is the combination of observations with physics-based models in order to provide the optimal estimate of the state of a system. In the context of short timescale core dynamics, progress in this direction is already being made in studies using the QG framework (Canet et al. 2009; Gillet et al. 2010). The application of data assimilation techniques to a wider range of forward models would allow the strengths and weaknesses of different models to be assessed in a powerful and direct manner. This could yield the quantitative tests of core dynamics hypotheses that have proven so elusive in the past.

Acknowledgements The authors wish to thank ISSI and in particular Andre Balogh for hosting the workshop on Terrestrial Magnetism in March 2009 that led to this review. Lively discussions with participants on a variety of topics related to short timescale core dynamics contributed much to this article. An anonymous reviewer as well as Nicolas Gillet, Alexandre Fournier and Elisabeth Canet are thanked for helpful comments 
on the manuscript. MD is currently supported by a Discovery grant from NSERC/CRSNG. This is IPGP contribution number 3067.

\section{References}

R. Abarca del Rio, D. Gambis, D.A. Salatein, Interannual signals in length of day and atmospheric angular momentum. Ann. Geophys. 18, 347-364 (2000)

D.J. Acheson, On hydromagnetic stability of a rotating fluid annulus. J. Fluid Mech. 52(3), 529-541 (1972)

D.J. Acheson, R. Hide, Hydromagnetics of rotating fluids. Rep. Prog. Phys. 36, 159-221 (1973)

H. Alfvén, On the existence of electromagnetic-hydromagnetic waves. Nature 150, 405-406 (1942)

D.W. Allan, E.C. Bullard, The secular variation of the Earth's magnetic field. Proc. Camb. Philos. Soc. 62(3), 783-809 (1966)

H. Amit, U.R. Christensen, Accounting for magnetic diffusion in core flow inversions from geomagnetic secular variation. Geophys. J. Int. 175, 913-924 (2008)

H. Amit, P. Olson, Helical core flows from geomagnetic secular variations. Phys. Earth Planet. Inter. 147, $1-25$ (2004)

H. Amit, P. Olson, Time-average and time-dependent parts of core flows. Phys. Earth Planet. Inter. 155, 120-139 (2006)

H. Amit, P. Olson, U. Christensen, Tests of core flow imaging methods with numerical dynamos. Geophys. J. Int. 168, 27-39 (2007)

H. Amit, J. Aubert, G. Hulot, P. Olson, A simple model for mantle-driven flow at the top of the Earth's core. Earth Planets Space 60, 845-854 (2008)

S. Asari, H. Shimizu, H. Utada, Robust and less robust features in the tangential geostrophy core flows. Geophys. J. Int. 178, 678-692 (2009)

J. Aubert, Steady zonal flows in spherical shell fluid dynamos. J. Fluid Mech. 542, 53-67 (2005)

J. Aubert, N. Gillet, P. Cardin, Quasigeostrophic models of convection in rotating spherical shells. Geochem. Geophys. Geosyst. 4, 1052 (2003). doi:10.1029/2002GC000456

J. Aubert, H. Amit, G. Hulot, Detecting thermal boundary control in surface flows from numerical dynamos. Phys. Earth Planet. Inter. 160, 143-156 (2007)

J. Aubert, J. Aurnou, J. Wicht, The magnetic structure of convection-driven numerical dynamos. Geophys. J. Int. 172, 945-966 (2008)

J. Aubert, J. Tarduno, C. Johnson, Observations and models of the long-term evolution of Earth's Magnetic Field. Space Sci. Rev. (2010). doi:10.1007/s11214-010-9684-5

G. Backus, Kinematics of geomagnetic secular variation in a perfectly conducting core. Philos. Trans. R. Soc. Lond. A 263, 239-266 (1968)

G. Backus, Bayesian inference in geomagnetism. Geophys. J. Int. 92, 125-142 (1988)

G.E. Backus, J.L. Le Mouël, The region on the core-mantle boundary where a geostrophic velocity field can be determined from frozen-flux magnetic data. Geophys. J. R. Astron. Soc. 85, 617-628 (1986)

E.R. Benton, K.A. Whaler, Rapid diffusion of the poloidal geomagnetic field through the weakly conducting mantle: a perturbation solution. Geophys. J. Int. 75, 77-100 (1983)

J. Bloxham, The expulsion of magnetic flux from the Earth's outer core. Geophys. J. R. Astron. Soc. 87, 669-678 (1986)

J. Bloxham, D. Gubbins, Geomagnetic field analysis-IV. Testing the frozen-flux hypothesis. Geophys. J. R. Astron. Soc. 84, 139-152 (1986)

J. Bloxham, A. Jackson, Lateral temperature variations at the core-mantle boundary deduced from the magnetic field. Geophys. Res. Lett. 17, 1997-2000 (1990)

J. Bloxham, A. Jackson, Fluid flow near the surface of Earth's outer core. Rev. Geophys. 29, 97-120 (1991)

J. Bloxham, A. Jackson, Time dependent mapping of the geomagnetic field at the core-mantle boundary. J. Geophys. Res. 97, 19537-19564 (1992)

J. Bloxham, D. Gubbins, A. Jackson, Geomagnetic secular variation. Philos. Trans. R. Soc. Lond. A 329(1606), 415-502 (1989)

J. Bloxham, S. Zatman, M. Dumberry, The origin of geomagnetic jerks. Nature 420, 65-68 (2002)

S.I. Braginsky, Magnetohydrodynamics of the Earth's core. Geomagn. Aeron. 4, 698-712 (1964)

S.I. Braginsky, Magnetic waves in the Earth's core. Geomagn. Aeron. 7, 851-859 (1967)

S.I. Braginsky, Torsional magnetohydrodynamic vibrations in the Earth's core and variations in day length. Geomagn. Aeron. 10, 1-10 (1970)

S.I. Braginsky, Analytic description of the geomagnetic field of past epochs and determination of the spectrum of magnetic waves in the core of the Earth I. Geomagn. Aeron. 12, 947-957 (1972)

S.I. Braginsky, Short-period geomagnetic secular variation. Geophys. Astrophys. Fluid Dyn. 30, 1-78 (1984) 
S.I. Braginsky, P.H. Roberts, Equations governing convection in Earth's core and the geodynamo. Geophys. Astrophys. Fluid Dyn. 79, 1-97 (1995)

B.A. Buffett, Free oscillations in the length of day: inferences on physical properties near the core-mantle boundary, in The Core-mantle Boundary Region, ed. by M. Gurnis, M.E. Wysession, E. Knittle, B.A. Buffett. Geodynamics Series, vol. 28 (AGU Geophysical Monograph, Washington, 1998), pp. 153165

B.A. Buffett, J. Mound, A. Jackson, Inversion of torsional oscillations for the structure and dynamics of Earth's core. Geophys. J. Int. 177, 878-890 (2009)

F.H. Busse, The dynamical coupling between inner core and mantle of the Earth and the 24-year libration of the pole, in Earthquake Displacement Fields and the Rotation of the Earth, ed. by D. Mansinha, D.E. Smylie, A.E. Beck. Astrophysics and Space Science Library, vol. 20 (Reidel, Dordrecht, 1970), pp. $88-98$

F.H. Busse, C. Carrigan, Laboratory simulation of thermal convection in rotating planets and stars. Science 191, 81-83 (1976)

F.H. Busse, R. Simitev, Convection in rotating spherical fluid shells and its dynamo states, in Fluid Dynamics and Dynamos in Astrophysics and Geophysics, ed. by A.M. Soward, C.A. Jones, D.W. Hugues, N.O. Weiss. The Fluid Mechanics of Astrophysics and Geophysics (Taylor \& Francis, London, 2005), pp. 359-392

E. Canet, Modèle dynamique et assimilation de données de la variation séculaire du champ magnétique terrestre. Ph.D. thesis, Université Joseph Fourier de Grenoble (2009)

E. Canet, A. Fournier, D. Jault, Forward and adjoint quasi-geostrophic models of the geomagnetic secular variation. J. Geophys. Res. 114 (2009). doi:10.1029/2008JB006189

P. Cardin, P. Olson, An experimental approach to thermochemical convection in the Earth's core. Geophys. Res. Lett. 19, 1995-1998 (1992)

P. Cardin, P. Olson, Chaotic thermal convection in a rapidly rotating spherical shell: consequences for flow in the outer core. Phys. Earth Planet. Inter. 82, 235-259 (1994)

C. Carrigan, F.H. Busse, An experimental and theoretical investigation of the onset of convection in rotating spherical shells. J. Fluid Mech. 126, 287-305 (1983)

S. Chandrasekhar, Hydrodynamic and Hydromagnetic Stability (Clarendon, Oxford, 1961), pp. 196-219

U.R. Christensen, J. Aubert, Scaling properties of convection-driven dynamos in rotating spherical shells and application to planetary magnetic fields. Geophys. J. Int. 166, 97-114 (2006)

U.R. Christensen, P. Olson, Secular variation in numerical geodynamo models with lateral variations of boundary heat flow. Geophys. J. Int. 138, 39-54 (2003)

U.R. Christensen, J. Wicht, Numerical dynamo simulations, in Treatise on Geophysics, vol. 8, ed. by P. Olson (Elsevier, Amsterdam, 2007), pp. 245-282

A. Chulliat, Geomagnetic secular variation generated by a tangentially geostrophic flow under the frozen-flux assumption-II. Sufficient conditions. Geophys. J. Int. 157, 537-552 (2004)

A. Chulliat, G. Hulot, Local computation of the geostrophic pressure at the top of the core. Phys. Earth Planet. Inter. 117, 309-328 (2000)

A. Chulliat, G. Hulot, Geomagnetic secular variation generated by a tangentially geostrophic flow under the frozen-flux assumption-I. Necessary conditions. Geophys. J. Int. 147, 237-246 (2001)

A. Chulliat, N. Olsen, Observation of magnetic diffusion in the Earth's outer core from Magsat, Oersted and CHAMP data. J. Geophys. Res. 115, B05105 (2010). doi:10.1029/2009JB006994

A. Chulliat, G. Hulot, L. Newitt, Magnetic flux expulsion from the core as a possible cause of the unusually large acceleration of the north magnetic pole during the 1990s. J. Geophys. Res. 115, B07101 (2010). doi: 10.1029/2009JB007143

C.G. Constable, R.L. Parker, P. Stark, Geomagnetic field models incorporating frozen-flux constraints. Geophys. J. Int. 113, 419-433 (1993)

P. Davidson, An Introduction to Magnetohydrodynamics (Cambridge University Press, Cambridge, 2001)

E. Dormy, A.M. Soward, C.A. Jones, D. Jault, Cardin, The onset of thermal convection in rotating spherical shells. J. Fluid Mech. 501, 43-70 (2004)

E. Dormy, P.H. Roberts, A.M. Soward, Core, boundary layers, in Encyclopedia of Geomagnetism and Paleomagnetism (Springer, Berlin, 2007)

S.J. Drew, Magnetic field expulsion into a conducting mantle. Geophys. J. Int. 115, 303-312 (1993)

M. Dumberry, Gravity variations induced by core flows. Geophys. J. Int. 180, 635-650 (2010)

M. Dumberry, J. Bloxham, Torque balance, Taylor's constraint and torsional oscillations in a numerical model of the geodynamo. Phys. Earth Planet. Inter. 140, 29-51 (2003)

M. Dumberry, J.E. Mound, Constraints on core-mantle electromagnetic coupling from torsional oscillation normal modes. J. Geophys. Res. 113, B03102 (2008). doi:10.1029/2007JB005135

A.M. Dziewonski, D.L. Anderson, Preliminary reference Earth model. Phys. Earth Planet. Inter. 25, $297-356$ (1981) 
C. Eymin, G. Hulot, On core surface flows inferred from satellite magnetic data. Phys. Earth Planet. Inter. 152, 200-220 (2005)

D.R. Fearn, Differential rotation and thermal convection in a rapidly rotating hydromagnetic system. Geophys. Astrophys. Fluid Dyn. 49, 173-193 (1989)

D.R. Fearn, Magnetic instabilities in rapidly rotating systems, in Theory of Solar and Planetary Dynamos, ed. by M.R.E. Proctor, P.C. Matthews, A.M. Rucklidge (1993), pp. 59-68

D.R. Fearn, Nonlinear planetary dynamos, in Lectures on Solar and Planetary Dynamos, ed. by M.R.E. Proctor, A.D. Gilbert (Cambridge University Press, Cambridge, 1994)

D.R. Fearn, Hydromagnetic flow in planetary cores. Rep. Prog. Phys. 61, 175-235 (1998)

C.C. Finlay, Waves in the presence of magnetic fields, rotation and convection. Les Houches Summer School Proc. 88, 403-450 (2008)

C.C. Finlay, A. Jackson, Equatorially dominated magnetic field change at the surface of Earth's core. Science 300, 2084-2086 (2003)

A. Fournier, G. Hulot, D. Jault, W. Kuang, A. Tangborn, N. Gillet, E. Canet, J. Aubert, F. Lhuillier, An introduction to data assimilation and predictability in geomagnetism. Space Sci. Rev. (2010, accepted). doi:10.1007/s11214-010-9669-4

E. Friis-Christensen, H. Lühr, G. Hulot, Swarm: A constellation to study the Earth's magnetic field. Earth Planets Space 58, 351-358 (2006)

H. Gellibrand, A Discourse Mathematical on the Variation of the Magnetic Needle. Together with Its Admirable Diminution Lately Discovered (William Jones, London, 1635)

A.D. Gilbert, Dynamo Theory, ed. by S. Friedlander, D. Serre. Handbook of Mathematical Fluid Dynamics, vol. 2 (Elsevier, New York, 2003), pp. 355-441

A.E. Gill, Atmosphere-Ocean Dynamics (Academic Press, San Diego, 1982)

N. Gillet, C.A. Jones, The quasi-geostrophic model for rapidly rotating spherical convection outside the tangent cylinder. J. Fluid Mech. 554, 343-369 (2006)

N. Gillet, V. Lesur, N. Olsen, Geomagnetic core field secular variation models. Space Sci. Rev. (2009a). doi:10.1007/s11214-009-9586-6

N. Gillet, A. Pais, D. Jault, Ensemble inversion of time-dependent core flow models. Geochem. Geophys. Geosyst. 10, Q06004 (2009b). doi:10.1029/2008GC002290

N. Gillet, D. Jault, E. Canet, A. Fournier, Fast torsional waves and strong magnetic field within the Earth's core. Nature 465, 764-777 (2010)

G.A. Glatzmaier, P.H. Roberts, A three-dimensional convective dynamo solution with rotating and finitely conducting inner core and mantle. Phys. Earth Planet. Inter. 91, 63-75 (1995)

H.P. Greenspan, The Theory of Rotating Fluids (Cambridge University Press, Cambridge, 1968)

D. Gubbins, Finding core motions from magnetic observations. Philos. Trans. R. Soc. Lond. A 306, 247-254 (1982)

D. Gubbins, Mechanism for geomagnetic polarity reversals. Nature 326, 167-169 (1987)

D. Gubbins, Dynamics of the secular variation. Phys. Earth Planet. Int. 68, 170-182 (1991)

D. Gubbins, A formalism for the inversion of geomagnetic data for core motions with diffusion. Phys. Earth Planet. Inter. 98, 193-206 (1996)

D. Gubbins, Geomagnetic constraints on stratification at the top of the Earth's core. Earth Planets Space 59, 661-664 (2007)

D. Gubbins, P. Kelly, A difficulty with using the frozen flux hypothesis to find steady core motions. Geophys. Res. Lett. 23, 1825-1828 (1996)

D. Gubbins, P.H. Roberts, Magnetohydrodynamics of the Earth's core. Geomagnetism 2, 1-183 (1987)

E. Halley, A theory of the variation of the magnetical compass. Philos. Trans. R. Soc. Lond. A 13, 208-221 (1683)

E. Halley, An account of the cause of the change of the variation of the magnetic needle; with an hypothesis of the structure of the internal part of the Earth. Philos. Trans. R. Soc. Lond. A 17, 563-578 (1692)

R. Hide, Free hydromagnetic oscillations of the Earth's core and the theory of geomagnetic secular variation. Philos. Trans. R. Soc. Lond. A 259, 615-647 (1966)

R. Hide, A note on short-term core-mantle coupling, geomagnetic secular variation impulses, and potential magnetic field invariants as Lagrangian tracers of core motions. Phys. Earth Planet. Int. 39, 297-300 (1985)

R. Hide, K. Stewartson, Hydromagnetic oscillations of the Earth's core. Rev. Geophys. Space Phys. 10, 579598 (1972)

R. Hide, D.H. Boggs, J.O. Dickey, Angular momentum fluctuations within the Earth's liquid core and torsional oscillations of the core-mantle system. Geophys. J. Int. 143, 777-786 (2000)

R.G. Hills, Convection in the Earth's mantle due to viscous shear at the core-mantle interface and due to large-scale buoyancy. Ph.D. thesis, New Mexico State University (1979)

R. Hollerbach, On the theory of the geodynamo. Phys. Earth Planet. Inter. 98, 163-185 (1996) 
R. Holme, Large-scale flow in the core, in Treatise on Geophysics, vol. 8, ed. by P. Olson (Elsevier, Amsterdam, 2007)

R. Holme, N. Olsen, Core surface flow modelling from high-resolution secular variation. Geophys. J. Int. 166, 518-528 (2006)

S.S. Hough, On the application of harmonic analysis to the dynamic theory of the Tides, part I. On Laplace's "oscillations of the first species" and on the dynamics of ocean currents. Philos. Trans. R. Soc. Lond. A 189, 201-257 (1897)

G. Hulot, A. Chulliat, On the possibility of quantifying diffusion and horizontal Lorentz forces at the Earth's core surface. Phys. Earth Planet. Inter. 135, 47-54 (2003)

G. Hulot, C. Eymin, B. Langlais, M. Mandea, N. Olsen, Small-scale structure of the geodynamo inferred from Oersted and Magsat satellite data. Nature 416, 620-623 (2002)

A. Jackson, Kelvin's theorem applied to the Earth's core. Proc. R. Soc. London, Ser. A 452, 2195-2201 (1996)

A. Jackson, Time-dependency of tangentially geostrophic core surface motions. Phys. Earth Planet. Inter. 103, 293-311 (1997)

A. Jackson, Intense equatorial flux spots on the surface of Earth's core. Nature 424, 760-763 (2003)

A. Jackson, C.C. Finlay, Geomagnetic secular variation and its applications to the core, in Treatise on Geophysics, vol. 5, ed. by G. Schubert (Elsevier, Amsterdam, 2007), pp. 147-193

A. Jackson, J. Bloxham, D. Gubbins, Time-dependent flow at the core surface and conservation of angular momentum in the coupled core-mantle system, in Dynamics of the Earth's Deep Interior and Earth Rotation, vol. 72, ed. by J.L. Le Mouël, D.E. Smylie, T. Herring (AGU Geophysical Monograph, Washington, 1993), pp. 97-107

A. Jackson, A.R.T. Jonkers, M.R. Walker, Four centuries of geomagnetic secular variation from historical records. Philos. Trans. R. Soc. Lond. A 358, 957-990 (2000)

A. Jackson, C.G. Constable, M.R. Walker, R.L. Parker, Models of Earth's main magnetic field incorporating flux and radial vorticity constraints. Geophys. J. Int. 171, 133-144 (2007)

D. Jault, Electromagnetic and topographic coupling, and lod variations, in Earth's Core and Lower Mantle, ed. by C.A. Jones, A. Soward, K. Zhang. The Fluid Mechanics of Astrophysics And Geophysics (Taylor \& Francis, London, 2003), pp. 56-76

D. Jault, Axial invariance of rapidly varying diffusionless motions in the Earth's core interior. Phys. Earth Planet. Inter. 166, 67-76 (2008)

D. Jault, J.L. Le Mouël, Physical properties at the top of the core and core surface motions. Phys. Earth Planet. Inter. 68, 76-84 (1991)

D. Jault, G. Légaut, Alfvén waves within the Earth's core, in Fluid Dynamics and Dynamos in Astrophysics and Geophysics, ed. by A.M. Soward, C.A. Jones, D.W. Hugues, N.O. Weiss. The Fluid Mechanics of Astrophysics and Geophysics (Taylor \& Francis, London, 2005), pp. 277-293

D. Jault, C. Gire, J.L. Le Mouël, Westward drift, core motions and exchanges of angular momentum between core and mantle. Nature 333, 353-356 (1988)

D. Jault, G. Hulot, J.L. Le Mouël, Mechanical core-mantle coupling and dynamo modelling. Phys. Earth Planet. Inter. 98, 187-191 (1996)

C.A. Jones, Dynamos in planets, in Stellar Astrophysical Fluid Dynamics, ed. by M. Thompson, J. Christensen-Dalsgaard (Cambridge University Press, Cambridge, 2003), pp. 159-178

C.A. Jones, Thermal and compositional convection in the outer core, in Treatise in Geophysics, Core Dynamics, vol. 8, ed. by P. Olson (Amsterdam, 2007), pp. 131-185

C.A. Jones, A.N. Soward, A.I. Mussa, The onset of convection in a rapidly rotating sphere. J. Fluid Mech. 405, 157-179 (2000)

C.A. Jones, A.I. Mussa, S.J. Worland, Magnetoconvection in a rapidly rotating sphere: the weak-field case. Proc. R. Soc. Lond. A 459, 773-797 (2003)

A. Kageyama, T. Miyagoshi, T. Satu, Formation of current coils in geodynamo simulations. Nature 454, 1106-1109 (2008)

R.R. Kerswell, Tidal excitation of hydromagnetic waves and their damping in the Earth. J. Fluid Mech. 274, 219-241 (1994)

M.D. Kohler, D.J. Stevenson, Modeling core fluid motions and the drift of magnetic field patterns at the CMB by use of topography obtained by seismic inversion. Geophys. Res. Lett. 17, 1473-1476 (1990)

J.L. Le Mouël, Outer core geostrophic flow and secular variation of Earth's geomagnetic field. Nature 311, 734-735 (1984)

J.L. Le Mouël, C. Gire, T. Madden, Motions at core surface in the geostrophic approximation. Phys. Earth Planet. Inter. 39, 270-287 (1985)

B. Lehnert, Magnetohydrodynamic waves under the action of the Coriolis force. Astrophys. J. 119, 647-654 (1954) 
V. Lesur, I. Wardinski, M. Rother, M. Mandea, GRIMM: the GFZ reference internal magnetic model based on vector satellite and observatory data. Geophys. J. Int. 173, 382-394 (2008)

V. Lesur, I. Wardinski, S. Asari, B. Minchev, M. Mandea, Modelling the Earth's core magnetic field under flow constraints. Earth Planets Space 62, 503-516 (2010)

P.W. Livermore, G. Ierley, A. Jackson, The structure of Taylor's constraint in three dimensions. Proc. R. Soc. Lond. A 464, 3149-3174 (2008)

P.W. Livermore, G. Ierley, A. Jackson, The construction of exact Taylor states. I: The full sphere. Geophys. J. Int. 179, 923-928 (2009)

J.J. Love, A critique of frozen-flux inverse modelling of a nearly steady geodynamo. Geophys. J. Int. 138, 353-365 (1999)

W.V.R. Malkus, Hydromagnetic planetary waves. J. Fluid Mech. 28(4), 793-802 (1967)

M. Mandea, R. Holme, A. Pais, A. Jackson, E. Qamili, Geomagnetic jerks: rapid core field variations and core dynamics (2010). doi:10.1007/s11214-010-9663-X

J. Matzka, A. Chulliat, M. Mandea, C. Finlay, E. Qamili, Direct observations from main field studies: from ground to space (2010). doi:10.1007/s11214-010-9693-4

S. Maus, On the applicability of the frozen flux approximation in core flow modelling as a function of temporal frequency and spatial degree. Geophys. J. Int. 175, 853-856 (2008)

T. Miyagoshi, A. Kageyama, T. Sato, Zonal flow formation in the Earth's core. Nature 463, 793-796 (2010)

H.K. Moffatt, Magnetic Field Generation in Electrically Conducting Fluids (Cambridge University Press, Cambridge, 1978)

V. Morin, E. Dormy, Time dependent $\beta$-convection in rapidly rotating spherical shell. Phys. Fluids 16, 16031609 (2004)

J.E. Mound, B.A. Buffett, Interannual oscillations in the length of day: implications for the structure of mantle and core. J. Geophys. Res. 108(B7), 2334 (2003). doi:10.1029/2002JB002054

J.E. Mound, B.A. Buffett, Mechanisms of core-mantle angular momentum exchange and the observed spectral properties of torsional oscillations. J. Geophys. Res. 110, 08103 (2005). doi:10.1029/2004JB003555

J.E. Mound, B.A. Buffett, Detection of a gravitational oscillation in length-of-day. Earth Planet. Sci. Lett. 243, 383-389 (2006)

H.C. Nataf, N. Gagnière, On the peculiar nature of turbulence in planetary dynamos. C. R. Phys. 9, 702-710 (2008)

M. Nornberg, H. Ji, E. Schartman, A. Roach, J. Goodman, Observation of magnetocoriolis waves in a liquid metal Taylor-Couette Experiment. Phys. Rev. Lett. 104, 074501 (2010)

M.S. O'Brien, C.G. Constable, R.L. Parker, Frozen-flux modelling for epochs 1915 and 1980. Geophys. J. Int. 128, 434-450 (1997)

N. Olsen, M. Mandea, Rapidly changing flows in the Earth's core. Nature Geosci. 1, 390-394 (2008)

N. Olsen, H. Lühr, T. Sabaka, M. Mandea, M. Rother, L. Tøffner-Clausen, S. Choi, CHAOS-A model of Earth's magnetic field derived from CHAMP ørsted and SAC-C magnetic satellite data. Geophys. J. Int. 166, 67-75 (2006)

N. Olsen, M. Mandea, T.J. Sabaka, L. Tøffner-Clausen, CHAOS-2-A geomagnetic field model derived from one decade of continuous satellite data. Geophys. J. Int. 142 (2009)

P. Olson, J. Aurnou, A polar vortex in the Earth's core. Nature 402, 170-173 (1999)

P. Olson, U.R. Christensen, The time-averaged magnetic field in numerical dynamos with non-uniform boundary heat flow. Geophys. J. Int. 151, 809-823 (2002)

P. Olson, U.R. Christensen, G.A. Glatzmaier, Numerical modeling of the geodynamo: mechanisms of field generation and equilibration. J. Geophys. Res. 104, 10383-10404 (1999)

A. Pais, G. Hulot, Length of day decade variations, torsional oscillations and inner core superrotation: evidence from recovered core surface zonal flows. Phys. Earth Planet. Inter. 118, 291-316 (2000)

M.A. Pais, D. Jault, Quasi-geostrophic flows responsible for the secular variation of the Earth's magnetic field. Geophys. J. Int. 173, 421-443 (2008)

M.A. Pais, O. Oliveira, F. Nogueira, Nonuniqueness of inverted core-mantle boundary flows and deviations from tangential geostrophy. J. Geophys. Res. 109, B08105 (2004). doi:10.1029/2004JB003012

J. Pedlosky, Geophysical Fluid Dynamics (Springer, New-York, 1987)

M.R.E. Proctor, Convection and magnetoconvection in a rapidly rotating sphere, in Lectures on Solar and Planetary Dynamos, ed. by M.R.E. Proctor, A.D. Gilbert (1994), pp. 97-115

J. Proudman, On the motions of solids in a liquid possessing vorticity. Proc. R. Soc. Lond. A 92, 408-424 (1916)

S. Rau, U.R. Christensen, A. Jackson, J. Wicht, Core flow inversion tested with numerical dynamo models. Geophys. J. Int. 141, 485-497 (2000)

P.H. Roberts, On the thermal instability of a self-gravitating fluid sphere containing heat sources. Philos. Trans. R. Soc. Lond. A 263, 93-117 (1968) 
P.H. Roberts, G.A. Glatzmaier, A test of the frozen-flux approximation using a new geodynamo model. Philos. Trans. R. Soc. Lond. A 358, 1109-1121 (2000)

P.H. Roberts, S. Scott, On analysis of the secular variation. J. Geomagn. Geoelectr. 17, 137-151 (1965)

P.H. Roberts, K. Stewartson, On finite amplitude convection in a rotating magnetic system. Philos. Trans. R. Soc. Lond. 277, 287-315 (1974)

T.J. Sabaka, N. Olsen, M.E. Purucker, Extending comprehensive models of the Earth's magnetic field with Ørsted and CHAMP data. Geophys. J. Int. 159, 521-547 (2004)

A. Sakuraba, P. Roberts, Generation of a strong magnetic field using uniform heat flux at the surface of the core. Nature Geosci. 2, 802-805 (2009)

N. Schaeffer, P. Cardin, Quasi-geostrophic model of the instabilities of the Stewartson layer in flat and depth varying containers. Phys. Fluids 17, 104111 (2005)

N. Schaeffer, P. Cardin, Quasi-geostrophic kinematic dynamos at low magnetic Prandtl number. Earth Planet. Sci. Lett. 245, 595-604 (2006)

D. Schmitt, Magneto-inertial waves in a rotating sphere. Geophys. Astrophys. Fluid Dyn. 104, 135-151 (2010)

D. Schmitt, T. Alboussière, D. Brito, P. Cardin, N. Gagnière, D. Jault, H.C. Nataf, Rotating spherical Couette flow in a dipolar magnetic field: experimental study of magneto-inertial waves. J. Fluid Mech. 604, 175-197 (2008)

B. Sreenivasan, C.A. Jones, Structure and dynamics of the polar vortex in the Earth's core. Geophys. Res. Lett. 32, L20301 (2005). doi:10.1029/2005GL023841

B. Sreenivasan, C.A. Jones, Azimuthal winds, convection and dynamo action in the polar regions of planetary cores. Geophys. Astrophys. Fluid Dyn. 100, 319-339 (2006)

F.D. Stacey, Core properties, physical, in Encyclopedia of Geomagnetism and Paleomagnetism, ed. by D. Gubbins, E. Herrero-Bervera (Springer, Dordrecht, 2007), pp. 91-94

F. Takahashi, M. Matsushima, Dynamo action in a rotating spherical shell at high Rayleigh numbers. Phys. Fluids 17, 076601 (2005)

F. Takahashi, M. Matsushima, Y. Honkura, Simulations of a quasi-Taylor state geomagnetic field including polarity reversals on the Earth simulator. Science 309, 459-461 (2005)

F. Takahashi, M. Matsushima, Y. Honkura, Scale variability in convection-driven mhd dynamos at low Ekman number. Phys. Earth Planet. Inter. 167, 168-178 (2008a)

F. Takahashi, H. Tsunakawa, M. Matsushima, N. Mochizuki, Y. Honkura, Effects of thermally heterogeneous structure in the lowermost mantle on geomagnetic field strength. Earth Planet. Sci. Lett. 272, 738-746 (2008b)

B.D. Tapley, M. Bettadpur, M. Watkins, C. Reigber, The gravity recovery and climate experiment: Mission overview and early results. Geophys. Res. Lett. 31, L09607 (2004). doi:10.1029/2004GL019920

G.I. Taylor, Motions of solids in fluid when the flow is not irrotational. Proc. R. Soc. Lond. A 93, 99-113 (1917)

G.I. Taylor, Experiments with rotating fluids. Proc. R. Soc. Lond. A 100, 114-124 (1921)

G.I. Taylor, Experiments on the motion of solid bodies in rotating fluids. Proc. R. Soc. Lond. A 104, 213-218 (1923)

J.B. Taylor, The magneto-hydrodynamics of a rotating fluid and the Earth's dynamo problem. Proc. R. Soc. Lond. A 274, 274-283 (1963)

A. Tilgner, F.H. Busse, Finite amplitude convection in rotating spherical fluid shells. J. Fluid Mech. 332, 359-376 (1997)

I. Wardinski, R. Holme, A time-dependent model of the Earth's magnetic field and its secular variation for the period 1980-2000. J. Geophys. Res. 111, B12101 (2006). doi:10.1029/2006JB004401

I. Wardinski, R. Holme, S. Asari, M. Mandea, The 2003 geomagnetic jerk and its relation to the core surface flows. Earth Planet. Sci. Lett. 267, 468-481 (2008)

K.A. Whaler, Does the whole of the Earth's core convect. Nature 287, 528-530 (1980)

J. Wicht, U.R. Christensen, Torsional oscillations in dynamo simulations. Geophys. J. Int. 181, 1367-1380 (2010)

A. Willis, B. Sreenivasan, D. Gubbins, Thermal core-mantle interaction: Exploring regimes for 'locked' dynamo action. Phys. Earth Planet. Inter. 165(1-2), 83-92 (2007). doi:10.1016/j.pepi.2007.08.002

S. Zatman, J. Bloxham, Torsional oscillations and the magnetic field within the Earth's core. Nature 388, 760-763 (1997)

K. Zhang, G. Schubert, Magnetohydrodynamics in rapidly rotating spherical systems. Ann. Rev. Fluid Mech. 32, 409-443 (2000)

K. Zhang, P. Earnshaw, X. Liao, F. Busse, On inertial waves in a rotating fluid sphere. J. Fluid Mech. 437, 103-119 (2001)

K. Zhang, X. Liao, G. Schubert, Nonaxisymmetric instabilities of a toroidal magnetic field in a rotating sphere. J. Fluid Mech. 585, 1124-1137 (2004) 